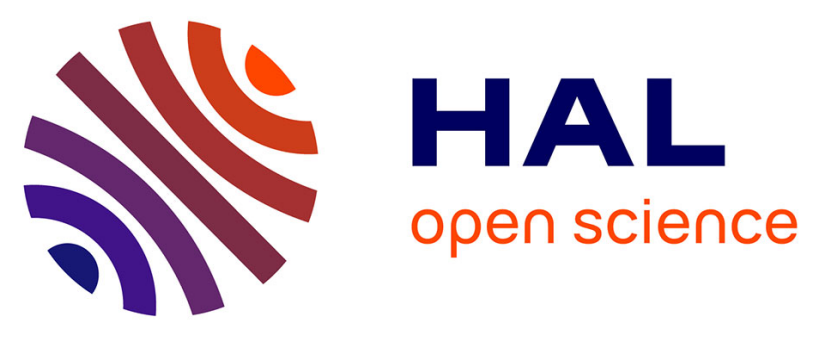

\title{
A Convenient Metal-Free Synthesis of (E)-3-Styrylisocoumarins through Annulation of (E)-1,4-Diarylenynes
}

Guangkuan Zhao, Ling-Zhi Yuan, Mylène Roudier, Jean-François Peyrat, Abdallah Hamze, Jean-Daniel Brion, Olivier Provot, Mouad Alami

\section{To cite this version:}

Guangkuan Zhao, Ling-Zhi Yuan, Mylène Roudier, Jean-François Peyrat, Abdallah Hamze, et al.. A Convenient Metal-Free Synthesis of (E)-3-Styrylisocoumarins through Annulation of (E)1,4-Diarylenynes. Synthesis: Journal of Synthetic Organic Chemistry, 2016, 48 (19), pp.3382-3392. 10.1055/s-0035-1562501 . hal-02394350

\section{HAL Id: hal-02394350 https://hal.science/hal-02394350}

Submitted on 6 Dec 2019

HAL is a multi-disciplinary open access archive for the deposit and dissemination of scientific research documents, whether they are published or not. The documents may come from teaching and research institutions in France or abroad, or from public or private research centers.
L'archive ouverte pluridisciplinaire HAL, est destinée au dépôt et à la diffusion de documents scientifiques de niveau recherche, publiés ou non, émanant des établissements d'enseignement et de recherche français ou étrangers, des laboratoires publics ou privés. 


\section{A convenient metal-free synthesis of (E)-3-styrylisocoumarins through annulation of $(E)$-1,4-diarylenynes}

\author{
Guangkuan. Zhao \\ Ling-Zhi Yuan ${ }^{\mathrm{a}}$ \\ Mylène Roudiera \\ Jean-François Peyrat ${ }^{a}$ \\ Abdallah Hamze \\ Jean-Daniel Briona \\ Olivier Provot ${ }^{\mathrm{*} *}$ \\ Mouad Alamia*
}

a BioCIS, Univ. Paris-Sud, CNRS, Université Paris-Saclay, 92290, Châtenay-Malabry, France.

olivier.provot@u-psud.fr

mouad.alami@u-psud.fr

This manuscript is dedicated to Professor Jean-François

Normant for his $80^{\text {th }}$ birthday

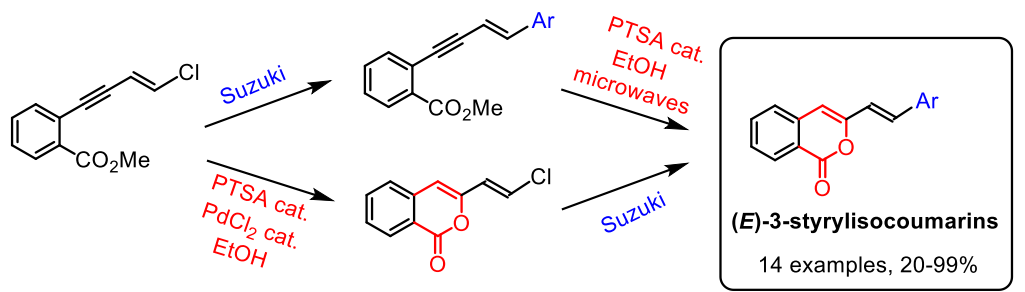

\section{Received:
Accepted:
Published onlin :}

Abstract: A metal-free procedure for the cyclization of (E)-1,4-diarylenynes is described. The reaction promoted by a catalytic amount of PTSA takes place in $\mathrm{EtOH}$ at $160{ }^{\circ} \mathrm{C}$ under microwaves irradiation and afforded stereoselectively (E)-3-styrylisocoumarins in good to excellent yields.

Key words styrylisocoumarins, cyclization, enynes, para-toluene sulfonic acid Suzuki couplings

Isocoumarins represent an important class of heteroaromatic compounds that exhibit a variety of pharmacological properties. ${ }^{1}$ In addition, they are endowed as a pivotal intermediate in the synthesis of several other heterocyclic structures such as isoquinolines and isochromenes. ${ }^{1}$ One of the most important subfamilies of isocoumarins is 3styrylisocoumarins, whose derivatives are the key structural units of a variety of natural products. ${ }^{2}$ While the synthesis of 3arylisocoumarins is well documented, 1,3 a careful examination of the literature reveals that general methods for the preparation of stereodefined 3-styrylisocoumarins remain under-developed.

Only three reports described the synthesis of 3-styrylisocoumarins, and within each work only one example is reported. Saeed ${ }^{4}$ and Rama $^{5}$ have disclosed the construction of polyoxygenated 3-styrylisocoumarins through the cyclocondensation of homophtalic acids with phenyl acrylate ${ }^{4}$ or phenylacryloyl chloride derivatives ${ }^{5}$ under extremely harsh conditions (Scheme 1). Recently, Kapur et al. ${ }^{6}$ have described, as

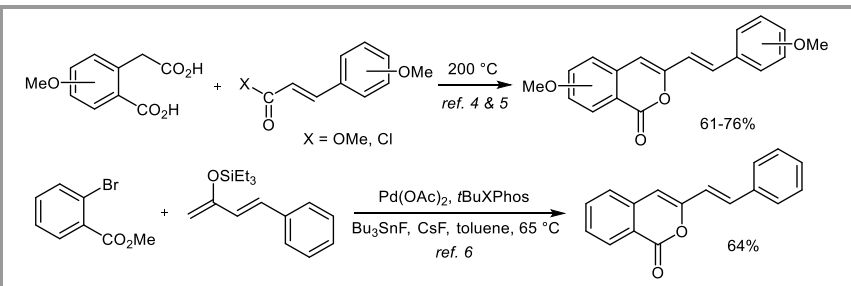

Scheme 1. Conventional strategies to 3-styryl-isocoumarins a single example, the Pd-catalyzed arylation of triethylsilylenol ethers of enones followed by a cyclization to provide the simple unfunctionalized 3-styrylisocoumarin. Therefore, there remains a need for the development of novel metal-free transformations tolerating a wide variety of substitution patterns on the styryl component, and proceeding with a high stereoselectivity.

As part of our medicinal chemistry program directed to the synthesis of heterocombretastatins, ${ }^{7}$ combined with our interest to the construction of novel substituted heterocycles through metal-free cyclization reactions, ${ }^{8}$ we required an efficient access to $(E)$-3-styryl-isocoumarins 1 containing several elements of structural diversity on the styryl unit. In our previous work, a metal-free annulation of diarylalkynes in the presence of a catalytic amount of $p$-toluenesulfonic acid (PTSA), under mild conditions, to afford a series of 3-arylisocoumarins has been successfully established. ${ }^{9}$ Inspired by these works, PTSAcatalyzed cyclization of alkyne-tethered olefin units bearing an aryl ester to synthesize targeted 3-styrylisocoumarins was proposed (Figure 1). The major challenge associated with this transformation is to achieve the cyclization reaction while keeping intact the stereochemistry of the $\mathrm{C}-\mathrm{C}$ double bond. Herein we report our success on the development of such a protocol that extends the scope of our previous work. ${ }^{9}$

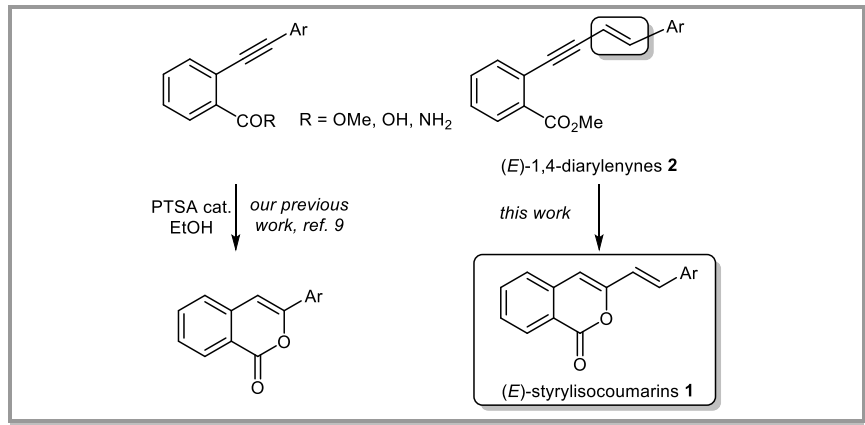

Figure 1 Access of $(E)$-3-styrylisocoumarins 1 from the cyclization of $(E)$-1,4diarylenynes 2 . 


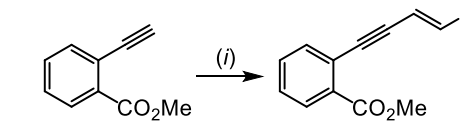

$(E)-\mathbf{4}$

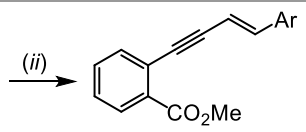

(E)-2a-n

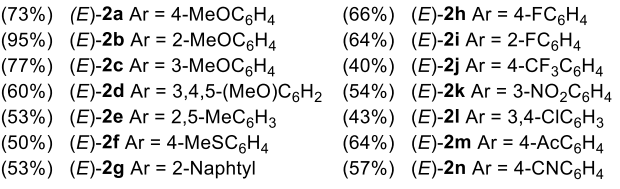

Scheme 2. Synthesis of $(E)-1,4$-diarylenynes $2 a-n$ from 3. Reagents and conditions: ( $i)(E)$-1,2-dichloroethylene (10 equiv), $\mathrm{PdCl}_{2}\left(\mathrm{PPh}_{3}\right)_{2}(10 \mathrm{~mol} \%), \mathrm{Cu}$ (10 mol\%), piperidine (2 equiv); $\mathrm{Et}_{2} \mathrm{O}$, rt. (ii) $\mathrm{ArB}(\mathrm{OH})_{2}$ (1.2 equiv), $\mathrm{Pd}\left(\mathrm{PPh}_{3}\right)_{4}(5$ mol\%), $\mathrm{K}_{2} \mathrm{CO}_{3}$ (2 equiv), toluene/ $\mathrm{MeOH}: 2 / 1$, reflux.

As depicted in Scheme 2, the required 1,4-diarylenyne derivatives 2a-n were synthesized in a two-step sequence from 2-methoxycarbonyl phenylacetylene 3. In details, Pd-catalyzed reaction with $(E)$-1,2-dichloroethylene and alkyne $\mathbf{3}$ afforded the desired (E)-chloroenyne $\mathbf{4}$ according to SonogashiraLinstrumelle coupling. ${ }^{10}$ Next, Suzuki coupling reactions ${ }^{11}$ between $(E)-\mathbf{4}$ and a variety of boronic acids was achieved, using $\mathrm{Pd}\left(\mathrm{PPh}_{3}\right)_{4}(5 \mathrm{~mol} \%)$ as a catalyst, $\mathrm{K}_{2} \mathrm{CO}_{3}$ as a base in a refluxing combination of toluene $\mathrm{MeOH}$ as a solvent. Accordingly, $(E)-1,4-$ diarylenynes $\mathbf{2}$ were formed stereo-selectively with satisfactory isolated yields (Scheme 2). Under these conditions, we were pleased to observe that the coupling process was efficient regardless the electronic nature of the boronic acids having electron-rich or electron-poor substituents in the ortho-, meta- or para-position.

We have next studied the cyclization of enyne $(E)$-2a into isocoumarin $(E)-\mathbf{1 a}$, as a model substrate to establish the best reaction conditions. The influence of several parameters were examined including, the solvent (EtOH, $\mathrm{MeOH}$ or $\mathrm{BnOH})$, the quantities of PTSA, and the reaction temperature (conventional or MW heating). The best conditions required the use of $30 \mathrm{~mol} \%$ of PTSA in EtOH and heating under microwave irradiation (MWI) at $160{ }^{\circ} \mathrm{C}$ to accelerate the reaction. Accordingly, isocoumarin $1 \mathrm{a}$ was formed in an almost a quantitative yield of $95 \%$ within $1.5 \mathrm{~h}$ (Table 1, entry 1). It should be noted that the optimal temperature was $160^{\circ} \mathrm{C}$, as lower temperatures resulted in a low conversion and inferior yields.

With this encouraging result in hand, we next turned our attention to examining the scope and limitations of this cyclization under the optimized reaction conditions. As shown in Table 1, we were able to transform a panel of $(E)-1,4-$ diarylenynes $\mathbf{2}$ bearing various functional groups into their corresponding isocoumarin derivatives 1. (E)-Enynes 2a-f bearing electron-donating substituents (OMe, $\mathrm{Me}$ or $\mathrm{SMe}$ ) on ortho-, meta- and para-positions on the aromatic nucleus were successfully transformed into the expected (E)-styrylisocoumarins 1a-f with satisfactory yields ranging from $63 \%$ to $90 \%$ (entries 1-6). A naphthyl aromatic ring was also well tolerated by the PTSA-catalyzed cyclization process and $2 \mathrm{~g}$ was transformed into isocoumarin $\mathbf{1 g}$ with a good $86 \%$ yield (entry 7). Enynes 2h-n with an electron-withdrawing group on the aromatic ring were next evaluated in the above cyclization methodology (entries 8-14). Altogether, the process was still efficient and led to the expected isocoumarins $\mathbf{1 h}-\mathbf{n}$ with yields ranging from $20 \%$ to $99 \%$, depending on the nature and the position of the electron-withdrawing substituent on the aromatic ring. It should be noted that the cyclization-reaction of enyne $\mathbf{2 n}$

Table 1. Synthesis of 3-styrylisocoumarins 1a-n

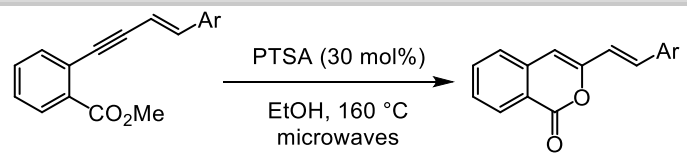

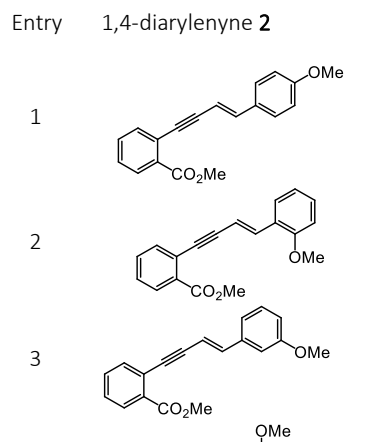

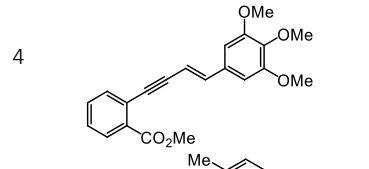<smiles>COc1ccc(/C=C/c2cc3ccccc3c(=O)o2)cc1</smiles><smiles>C=CC=CC=CC=CC=Cc1ccc(C)cc1</smiles>

7<smiles>COC(=O)c1ccccc1CC=CC=Cc1ccc2ccccc2c1</smiles><smiles>COc1ccccc1/C=C/c1cc2ccccc2c(=O)o1</smiles><smiles>COc1cccc(/C=C/c2cc3ccccc3c(=O)o2)c1</smiles>

(E)-1c 63<smiles>COc1cc(/C=C/c2cc3ccccc3oc2=O)cc(OC)c1OC</smiles>

(E)-1e 77
10<smiles>COC(=O)c1ccccc1C#CC=Cc1ccc(C(F)(F)F)cc1</smiles>

11<smiles>COC(=O)c1ccccc1C=CC=Cc1cccc([N+](=O)[O-])c1</smiles>

12<smiles>COc1ccccc1C#CC=Cc1ccc(Cl)c(Cl)c1</smiles>

13<smiles>COC(=O)c1ccccc1CC=Cc1ccc(Cl)cc1</smiles>

14

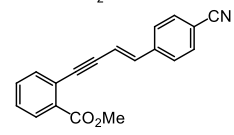

a Yield of isolated product.
Yield $\%)^{\mathrm{a}}$

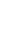

(7)

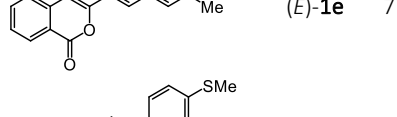

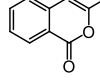<smiles>O=c1oc(C=Cc2ccc3ccccc3c2)cc2ccccc12</smiles><smiles>O=c1oc(/C=C/c2ccc(F)cc2)cc2ccccc12</smiles><smiles>O=c1oc(/C=C/c2ccccc2F)cc2ccccc12</smiles><smiles></smiles><smiles>O=c1oc(/C=C/c2cccc([N+](=O)[O-])c2)cc2ccccc12</smiles><smiles></smiles>

(E)-11 67<smiles>N#Cc1ccc(/C=C/c2cc3ccccc3c(=O)o2)cc1/C=C/c1ccc(Cl)cc1</smiles>

(E)$1 \mathrm{~m}$

gave isocoumarin 1n but with a low 20\% yield (entry 14), probably because the $\mathrm{CN}$-function of $\mathbf{2 n}$ reacted with PTSA, as we had previously reported with other $\mathrm{CN}$-containing substrates under similar conditions. ${ }^{9}$

We next focused our attention whether the developed protocol would be applicable with (Z)-1,4-diarylenynes to provide the corresponding (Z)-3-styrylisocoumarins. A Pdcoupling reaction between alkyne $\mathbf{3}$ and (Z)-1,2-dichloroethylene in the presence of $\mathrm{PdCl}_{2}\left(\mathrm{PPh}_{3}\right)_{2}, \mathrm{CuI}$, and $n$-butylamine 


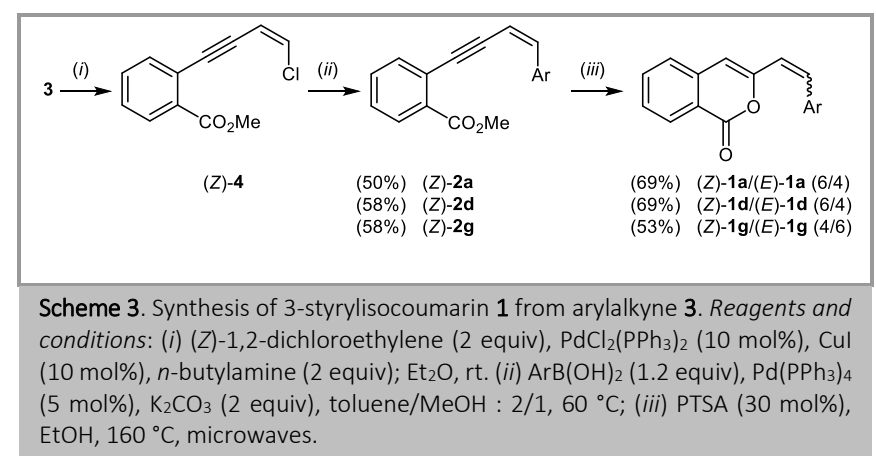

afforded stereoselectively the (Z)-chloroenyne $\mathbf{4}^{10}$ in a $52 \%$ yield (Scheme 3). Subsequent Suzuki-coupling reactions furnished selectively the expected $(Z)$-1,4-diarylenynes $(Z)-2 \mathbf{a},(Z)-\mathbf{2 d},(Z)$ $2 \mathrm{~g}$ with no trace of isomerization of their $(Z)$-double bond. When 1,4-diarylenynes $(Z)$-2 were treated under the optimized reaction conditions, we were pleased to observe that the cyclization reaction was still efficient but led to a mixture of nonseparable $Z / E$ isocoumarins $\mathbf{1}$ in nearly equivalent proportions (Scheme 3).

On the basis of the results depicted in Table 1, we believe that this annulation process proceeds as shown in Scheme 4 by a sequence involving ( $i$ ) activation of the triple bond by PTSA, (ii) nucleophilic attack by the oxygen of the ester group to the more electrophilic acetylenic carbon according to a favored 6-endo-dig process, ${ }^{12}$ and (iii) loss of the methyl group of the oxonium species promoted by the solvent ${ }^{8 b}$ to afford the 3styrylisocoumarin 1 together with one equivalent of EtOMe.

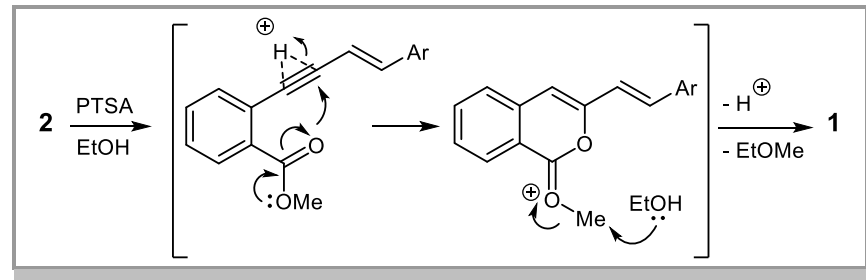

Scheme 4. Proposed mechanism for the PTSA-catalyzed annulation of 2

We next examined the possibility of preparing (E)-3-styrylisocoumarins $\mathbf{1}$ from isocoumarin $\mathbf{5}$ bearing a vinyl chloride function on the 3-position. Accordingly, the PTSA-catalyzed annulation of $(E)$-chloroenyne $\mathbf{4}$ was investigated. Thus, heating (E)-4 under our optimized conditions (EtOH, $160{ }^{\circ} \mathrm{C}, \mathrm{MWI}, 1 \mathrm{~h}$ ) yielded the expected isocoumarin $(E)-5$ in a $60 \%$ yield (Table 2 , entry 1). It should be noted that achieving the annulation under conventional heating $\left(\mathrm{EtOH}, 78^{\circ} \mathrm{C}\right)$ required longer reaction time $(24 \mathrm{~h})$ and gave $(E)-\mathbf{5}$ in a lower yield (30\%, entry 2$)$. One notes that little improvement was realized with increasing the amount of PTSA (entry 3). The effects of Lewis acids (e.g., $\mathrm{FeBr}_{3}, \mathrm{InCl}_{3}$, $\mathrm{PdCl}_{2}$ ) were then briefly studied in refluxing EtOH or under microwave irradiation (entries 4-7). No improvement in yield was observed using these metal catalysts; the best result $(50 \%$, entry 7) was obtained using a catalytic amount of $\mathrm{PdCl}_{2}(10$ mol\%) after refluxing in $\mathrm{EtOH}$ for a long reaction time of $30 \mathrm{~h}$.

By combining the use of catalytic amounts of $\mathrm{PdCl}_{2}$ (10 mol\%) and PTSA (10 mol\%) in refluxing EtOH for $24 \mathrm{~h}$, we were pleased to observe that $(E)$-chloroenyne $\mathbf{4 a}$ undergo the annulation process successfully providing the expected isocoumarin $\mathbf{5}$ with a good $70 \%$ yield (entry 8 ).
Table 2. Effect of additives mediated cyclization of (E)-chloroenyne $4 a$<smiles>C/C=C/C#Cc1ccccc1C(=O)OC</smiles>

(E)-4

Entry Reactants

PTSA (10 mol\%), MWI, $160^{\circ} \mathrm{C}, 1 \mathrm{~h}$

PTSA (10 mol\%), reflux, $24 \mathrm{~h}$

PTSA (50 mol\%), reflux, $24 \mathrm{~h}$

$\mathrm{FeBr}_{3}(20$ mol\%), reflux, $15 \mathrm{~h}$

$\mathrm{FeBr}_{3}(20 \mathrm{~mol} \%), \mathrm{MWI}, 160^{\circ} \mathrm{C}, 1 \mathrm{~h}$

$\mathrm{PdCl}_{2}(10$ mol\%), reflux, $30 \mathrm{~h}$

$\mathrm{PdCl}_{2}$ (10 mol\%), PTSA (10 mol\%), reflux, $24 \mathrm{~h}$

$\mathrm{PdCl}_{2}$ (30 mol\%), PTSA (30 mol\%), reflux, $1 \mathrm{~h}$

a Yield of isolated product. ${ }^{\mathrm{b}} \mathrm{Nd}$ not determined.
$\mathrm{InCl}_{3}(20 \mathrm{~mol} \%)$, reflux, $30 \mathrm{~h}$

Finally, we found that increasing the quantities of PTSA and $\mathrm{PdCl}_{2}$ (from $10 \%$ to $30 \%$ ) allowed to prepare 3(chlorovinyl)isocoumarin $\mathbf{5}$ in a good $75 \%$ yield after $1 \mathrm{~h}$ refluxing in EtOH (entry 9). By applying these experimental conditions to the chloroenyne (Z)-4 (Scheme 5), we have observed the formation of the expected (Z)-3(chlorovinyl)isocoumarin $\mathbf{5}$ but with a very low yield of $15 \%$ accompanied by its $(E)$-isomer 5 (21\%).

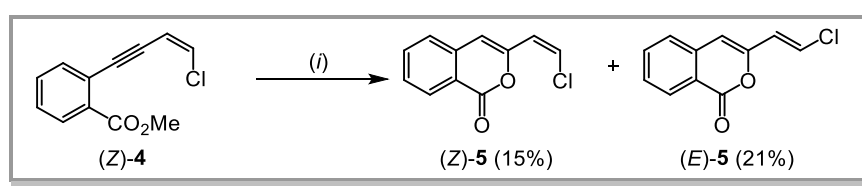

Scheme 5 . Synthesis of (Z)-3-(chlorovinyl)isocoumarin 5 by cyclization of (Z)chloroenyne 4. Reagents and conditions: (i) $\mathrm{PdCl}_{2}$ (30 mol\%), PTSA (30 mol\%), EtOH reflux, $1 \mathrm{~h}$.

With $(E)-5$ in hand, its Suzuki coupling with boronic acids under the conditions described in Scheme 2, however, failed, clearly demonstrating that the $\mathrm{C}_{\mathrm{sp} 2}-\mathrm{Cl}$ bond of chloroenyne $\mathbf{4}$ and 3-(chlorovinyl)isocoumarin $\mathbf{5}$ displayed different reactivity. After several attempts, we found that the protocol ${ }^{13}$ involving the use of $\mathrm{Pd}_{2} \mathrm{dba}_{3} . \mathrm{CHCl}_{3}$ as the catalyst, and $\mathrm{P}(t-\mathrm{Bu})_{3}$ as the ligand in the presence of $\mathrm{KF}$ as the base proved to be suitable furnishing the desired (E)-3-styrylisocoumarin 1a in a good $73 \%$ yield (Scheme 6).

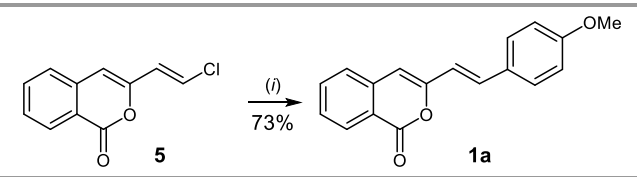

Scheme 6. Synthesis of (E)-1a from (E)-3-(chlorovinyl)isocoumarin 5. Reagents and conditions: (i) 4-methoxyphenylboronic acid (4 equiv), KF (3.3 equiv), $\mathrm{Pd}_{2} \mathrm{dba}_{3} . \mathrm{CHCl}_{3}(5 \mathrm{~mol} \%), \mathrm{P}(t-\mathrm{Bu})_{3}(10 \mathrm{~mol} \%), \mathrm{THF}, 65^{\circ} \mathrm{C}, 7 \mathrm{~h}$.

In summary, we demonstrated that chloroenynes of type 4 constitutes a common starting material for the synthesis of various 3-styrylisocoumarins $\mathbf{1}$ having several elements of structural diversity on the styryl unit. The strategy developed is general and involves two different and complementary pathways: (i) a Suzuki coupling reaction of boronic acids with (E)-chloroenynes of type $\mathbf{4}$ followed by cyclization in the presence of catalytic amounts of PTSA, or (ii) a PTSA/ $\mathrm{PdCl}_{2}-$ catalyzed the cyclization of $(E)$-chloroenynes $\mathbf{4}$ followed by Suzuki couplings on the resulting 3-chlorovinylisocoumarin $\mathbf{5}$. Some of these new derivatives $\mathbf{1}$, having a great resemblance to biologically active heteroaromatic analogs ${ }^{14}$ of combretastatins 
will be evaluated later in our lab for their possible antitumor activities.

All glasswares were oven-dried at $140{ }^{\circ} \mathrm{C}$ and all reactions were conducted under a nitrogen atmosphere. Solvents: cyclohexane, ethyl acetate (EtOAc), for chromatography, were technical grade. All new compounds were characterized by ${ }^{1} \mathrm{H}-\mathrm{NMR},{ }^{13} \mathrm{C}-\mathrm{NMR}$, IR spectroscopy and HRMS. ${ }^{1} \mathrm{H}$ and ${ }^{13} \mathrm{C}$ NMR spectra were measured in $\mathrm{CDCl}_{3}$ with a Bruker Avance 300. ${ }^{1} \mathrm{H}$ chemical shifts are reported in ppm from an internal standard TMS or of residual chloroform (7.26 ppm). The following abreviation are used: $m$ (multiplet), s (singlet), d (doublet), t (triplet), dd (doublet of doublet), td (triplet of doublet), ddd (doublet of doublet of doublet). ${ }^{13} \mathrm{C}$ chemical shifts are reported in ppm from the central peak of deuteriochloroform (77.14). IR spectra were measured on a Bruker Vector 22 spectrophotometer (neat, $\mathrm{cm}^{-1}$ ). High-resolution mass spectra were recorded on a Bruker Daltonics micrOTOF-Q instrument. Analytical TLC was performed on Merck precoated silica gel 60 F-254 plates. Merck silica gel 60 (230-400 mesh) was used for column chromatography. The plates were visualized by either UV light (254 $\mathrm{nm}$ ), or by a solution of phosphomolybdic acid in ethanol.

\section{Methyl (E)-2-(4-chlorobut-3-en-1-yn-1-yl)benzoate 4 (55\%)}

To a solution of alkyne 3 (160 mg, $1 \mathrm{mmol})$ in $\mathrm{Et}_{2} \mathrm{O}(15 \mathrm{~mL})$ was added successively (E)-1,2-dichloroethylene (0.8 mL, $10 \mathrm{mmol}), \mathrm{PdCl}_{2}\left(\mathrm{PPh}_{3}\right)_{2}$ (70 mg, $0.1 \mathrm{mmol})$, piperidine $(0.2 \mathrm{~mL}, 2 \mathrm{mmol}$ ) and CuI by portions (19 $\mathrm{mg}, 0.1 \mathrm{mmol}$ ). After $7 \mathrm{~h}$ of stirring at $\mathrm{rt}$, the solution was filtered on a pad of $\mathrm{SiO}_{2}$ using EtOAc. Organic layer was successively washed with a saturated $\mathrm{NH}_{4} \mathrm{Cl}$, saturated $\mathrm{NaHCO}_{3}$ and $\mathrm{HCl} 1 \mathrm{M}$ solutions. After drying on $\mathrm{Na}_{2} \mathrm{SO}_{4}$ and evaporation in vacuo, the crude was purified by chromatography on $\mathrm{SiO}_{2}$ to give 4 (121 $\mathrm{mg}, 0.55 \mathrm{mmol}$ ).

IR (neat): 3466, 2339, 2219, 2151, 2104, 1974, 1936, $1727 \mathrm{~cm}^{-1}$.

${ }^{1} \mathrm{H} \mathrm{NMR}\left(300 \mathrm{MHz}, \mathrm{CDCl}_{3}\right) \delta=7.95(\mathrm{dd}, J=7.8 \mathrm{~Hz}, J=1.0 \mathrm{~Hz}, 1 \mathrm{H}), 7.54(\mathrm{dd}$, $J=7.8 \mathrm{~Hz}, J=1.0 \mathrm{~Hz}, 1 \mathrm{H}), 7.46(\mathrm{td}, J=7.5 \mathrm{~Hz}, J=1.2 \mathrm{~Hz}, 1 \mathrm{H}), 7.37(\mathrm{td}, J=$ $7.5 \mathrm{~Hz}, J=1.2 \mathrm{~Hz}, 1 \mathrm{H}), 6.68(\mathrm{~d}, J=13.6 \mathrm{~Hz}, 1 \mathrm{H}), 6.22(\mathrm{~d}, J=13.6 \mathrm{~Hz}, 1 \mathrm{H})$, $3.92(\mathrm{~s}, 3 \mathrm{H})$.

${ }^{13} \mathrm{C}$ NMR (75 MHz, $\left.\mathrm{CDCl}_{3}\right) \delta=166.3(\mathrm{Cq}), 134.0(\mathrm{CH}), 131.8(\mathrm{CH}), 131.6$ $(\mathrm{Cq}), 130.9(\mathrm{CH}), 130.5(\mathrm{CH}), 128.2(\mathrm{CH}), 123.2(\mathrm{Cq}), 114.0(\mathrm{CH}), 90.6(\mathrm{Cq})$, $89.3(\mathrm{Cq}), 52.2\left(\mathrm{CH}_{3}\right)$.

HRMS Calcd for $\mathrm{C}_{12} \mathrm{H}_{10} \mathrm{O}_{2} \mathrm{Cl}[\mathrm{M}+\mathrm{H}]^{+} 221.0369$, found 221.0363.

\section{Methyl (Z)-2-(4-chlorobut-3-en-1-yn-1-yl)benzoate 4 (52\%)}

The protocol was identical to that developed for $(E)-4$ but $n \mathrm{BuNH}_{2}(200$ $\mu \mathrm{L} \mathrm{mg}, 2 \mathrm{mmol})$ replaced piperidine and $800 \mu \mathrm{L}(20 \mathrm{mmol})$ of $(Z)-1,2$ dichloroethylene were used. The crude was purified by chromatography on $\mathrm{SiO}_{2}$ to give 4 (114 mg, $0.52 \mathrm{mmol}$ )

IR (neat): $3087,2951,1729,1711,1582,1565,1484,1447,1432,1335$, $1294,1277,1252,1190,1164,1128,1083,1043,962,831,801,756,724$, $697,660,634 \mathrm{~cm}^{-1}$.

${ }^{1} \mathrm{H} \mathrm{NMR}\left(300 \mathrm{MHz}, \mathrm{CDCl}_{3}\right) \delta=7.95(\mathrm{~d}, J=7.8 \mathrm{~Hz}, 1 \mathrm{H}), 7.61(\mathrm{~d}, J=7.8 \mathrm{~Hz}$, $1 \mathrm{H}), 7.47(\mathrm{td}, J=7.6 \mathrm{~Hz}, J=1.2 \mathrm{~Hz}, 1 \mathrm{H}), 7.37(\mathrm{td}, J=7.6 \mathrm{~Hz}, J=1.2 \mathrm{~Hz}, 1 \mathrm{H})$, $6.47(\mathrm{~d}, J=7.5 \mathrm{~Hz}, 1 \mathrm{H}), 6.16(\mathrm{~d}, J=7.5 \mathrm{~Hz}, 1 \mathrm{H}), 3.93(\mathrm{~s}, 3 \mathrm{H})$.

${ }^{13} \mathrm{C}$ NMR $\left(75 \mathrm{MHz}, \mathrm{CDCl}_{3}\right) \delta=166.6(\mathrm{Cq}), 134.4(\mathrm{CH}), 131.8(\mathrm{Cq}), 131.7$ $(\mathrm{CH}), 130.4(\mathrm{CH}), 128.8(\mathrm{CH}), 128.4(\mathrm{CH}), 123.1(\mathrm{Cq}), 112.3(\mathrm{CH}), 96.0$ (Cq), $88.0(\mathrm{Cq}), 52.3\left(\mathrm{CH}_{3}\right)$.

HRMS Calcd for $\mathrm{C}_{12} \mathrm{H}_{10} \mathrm{O}_{2} \mathrm{Cl}[\mathrm{M}+\mathrm{H}]+221.0369$, found 221.0363 .

\section{General procedure for the synthesis of diarylenynes 2}

To a solution of 4 (220 mg, $1 \mathrm{mmol})$ in toluene/MeOH: 2/1 (10 mL) were added the boronic acid (1.2 mmol), $\mathrm{K}_{2} \mathrm{CO}_{3}(373 \mathrm{mg}, 2 \mathrm{mmol})$ and
$\mathrm{Pd}\left(\mathrm{PPh}_{3}\right)_{4}$ (58 $\left.\mathrm{mg}, 0.05 \mathrm{mmol}\right)$. The mixture was stirred under reflux until the disappearance of $\mathbf{4}$ (judged by TLC). After concentration, the crude was diluted in $\mathrm{CH}_{2} \mathrm{Cl}_{2}$ and washed with water. After drying on $\mathrm{Na}_{2} \mathrm{SO}_{4}$ and evaporation in vacuo, the crude was purified by chromatography on $\mathrm{SiO}_{2}$.

Methyl (E)-2-(4-(4-methoxyphenyl)but-3-en-1-yn-1-yl)benzoate 2a (Yield: $213 \mathrm{mg}, 0.73 \mathrm{mmol}, 73 \%$ )

IR (neat): 3033, 3001, 2951, 2837, 2360, 2192, 1729, 1603, 1563, 1511, $1483,1462,1445,1433,1343,1308,1289,1243,1174,1128,1084,1031$, $954,848,815,757,735,699,658 \mathrm{~cm}^{-1}$.

${ }^{1} \mathrm{H}$ NMR (300 MHz, $\left.\mathrm{CDCl}_{3}\right) \delta=7.97(\mathrm{~d}, J=7.8 \mathrm{~Hz}, 1 \mathrm{H}), 7.60(\mathrm{~d}, J=7.8 \mathrm{~Hz}$, $1 \mathrm{H}), 7.48(\mathrm{t}, J=7.0 \mathrm{~Hz}, 1 \mathrm{H}), 7.33-7.42(\mathrm{~m}, 3 \mathrm{H}), 7.07(\mathrm{~d}, J=16.0 \mathrm{~Hz}, 1 \mathrm{H})$, $6.90(\mathrm{~d}, J=8.7 \mathrm{~Hz}, 2 \mathrm{H}), 6.34(\mathrm{~d}, J=16.0 \mathrm{~Hz}, 1 \mathrm{H}), 3.98\left(\mathrm{~s}, 3 \mathrm{H}, \mathrm{CH}_{3}\right), 3.85$ (s, $\left.3 \mathrm{H}, \mathrm{CH}_{3}\right)$.

${ }^{13} \mathrm{C}$ NMR (75 MHz, $\left.\mathrm{CDCl}_{3}\right) \delta=160.2(\mathrm{Cq}), 141.6(\mathrm{CH}), 134.0(\mathrm{CH}), 131.7$ $(\mathrm{CH}), 131.4(\mathrm{Cq}), 130.4(\mathrm{CH}), 129.2(\mathrm{Cq}), 127.8(2 \mathrm{CH}), 127.6(\mathrm{CH}), 124.2$ $(\mathrm{Cq}), 114.2(2 \mathrm{CH}), 105.9(\mathrm{CH}), 94.5(\mathrm{Cq}), 89.8(\mathrm{Cq}), 55.3\left(\mathrm{CH}_{3}\right), 52.2\left(\mathrm{CH}_{3}\right)$, one $\mathrm{C}$ not seen.

HRMS Calcd for $\mathrm{C}_{19} \mathrm{H}_{17} \mathrm{O}_{3}[\mathrm{M}+\mathrm{H}]^{+}$293.1178, found 293.1178 .

Methyl (E)-2-(4-(2-methoxyphenyl)but-3-en-1-yn-1-yl)benzoate 2b (Yield: 277 mg, $0.95 \mathrm{mmol}, 95 \%$ )

IR (neat): 3439, 3153, 2839, 2359, 2005, 1729, $1711 \mathrm{~cm}^{-1}$.

${ }^{1} \mathrm{H}$ NMR $\left(300 \mathrm{MHz}, \mathrm{CDCl}_{3}\right) \delta=7.90(\mathrm{dd}, J=7.8 \mathrm{~Hz}, J=1.0 \mathrm{~Hz}, 1 \mathrm{H}), 7.52(\mathrm{~d}$, $J=7.5 \mathrm{~Hz}, 1 \mathrm{H}), 7.42-7.36(\mathrm{~m}, 2 \mathrm{H}), 7.30-7.17(\mathrm{~m}, 3 \mathrm{H}), 6.90-6.80(\mathrm{~m}$, $2 \mathrm{H}), 6.47(\mathrm{~d}, J=16.2 \mathrm{~Hz}, 1 \mathrm{H}), 3.89(\mathrm{~s}, 3 \mathrm{H}), 3.81(\mathrm{~s}, 3 \mathrm{H})$.

${ }^{13} \mathrm{C}$ NMR $\left(75 \mathrm{MHz}, \mathrm{CDCl}_{3}\right) \delta=166.7(\mathrm{Cq}), 157.1(\mathrm{Cq}), 137.4(\mathrm{CH}), 134.0$ $(\mathrm{CH}), 131.6(\mathrm{CH}), 131.5(\mathrm{Cq}), 130.4(\mathrm{CH}), 129.8(\mathrm{CH}), 127.6(\mathrm{CH}), 127.0$ $(\mathrm{CH}), 125.3(\mathrm{Cq}), 124.2(\mathrm{Cq}), 120.7(\mathrm{CH}), 111.1(\mathrm{CH}), 108.8(\mathrm{CH}), 94.9(\mathrm{Cq})$, $90.0(\mathrm{Cq}), 55.5\left(\mathrm{CH}_{3}\right), 52.2\left(\mathrm{CH}_{3}\right)$.

HRMS Calcd for $\mathrm{C}_{19} \mathrm{H}_{17} \mathrm{O}_{3}[\mathrm{M}+\mathrm{H}]+2$ 293.1178, found 293.1169 .

Methyl (E)-2-(4-(3-methoxyphenyl)but-3-en-1-yn-1-yl)benzoate 2c (Yield: $225 \mathrm{mg}, 0.77 \mathrm{mmol}, 77 \%$ )

IR (neat): 2950, 2836, 2194, 1729, 1711, 1596, 1578, 1483, 1432, 1292, $1274,1252,1191,1156,1134,1086,1046,952,827,783,757,687 \mathrm{~cm}^{-1}$.

${ }^{1} \mathrm{H}$ NMR (300 MHz, $\left.\mathrm{CDCl}_{3}\right) \delta=7.96(\mathrm{~d}, J=7.8 \mathrm{~Hz}, 1 \mathrm{H}), 7.59(\mathrm{~d}, J=7.8 \mathrm{~Hz}$, $1 \mathrm{H}), 7.47(\mathrm{td}, J=7.5 \mathrm{~Hz}, J=1.0 \mathrm{~Hz}, 1 \mathrm{H}), 7.36(\mathrm{~d}, J=7.5 \mathrm{~Hz}, 1 \mathrm{H}), 7.26(\mathrm{t}, J=$ $7.8 \mathrm{~Hz}, 1 \mathrm{H}), 7.09-7.03(\mathrm{~m}, 2 \mathrm{H}), 6.97(\mathrm{~s}, 1 \mathrm{H}), 6.86(\mathrm{dd}, J=8.2 \mathrm{~Hz}, J=2.5 \mathrm{~Hz}$, $1 \mathrm{H}), 6.46(\mathrm{~d}, J=16.2 \mathrm{~Hz}, 1 \mathrm{H}), 3.96(\mathrm{~s}, 3 \mathrm{H}), 3.83(\mathrm{~s}, 3 \mathrm{H})$.

${ }^{13} \mathrm{C}$ NMR $\left(75 \mathrm{MHz}, \mathrm{CDCl}_{3}\right) \delta=166.6(\mathrm{Cq}), 159.9(\mathrm{Cq}), 141.8(\mathrm{CH}), 137.7$ (Cq), $134.1(\mathrm{CH}), 131.7(\mathrm{CH}), 131.5(\mathrm{Cq}), 130.4(\mathrm{CH}), 129.7(\mathrm{CH}), 127.8$ $(\mathrm{CH}), 124.0(\mathrm{Cq}), 119.2(\mathrm{CH}), 114.5(\mathrm{CH}), 111.6(\mathrm{CH}), 108.6(\mathrm{CH}), 93.9$ (Cq), $90.6(\mathrm{Cq}), 55.3\left(\mathrm{CH}_{3}\right), 52.2\left(\mathrm{CH}_{3}\right)$.

HRMS Calcd for $\mathrm{C}_{19} \mathrm{H}_{16} \mathrm{O}_{3} \mathrm{Na}[\mathrm{M}+\mathrm{Na}]^{+}$315.0997, found 315.0993.

\section{Methyl (E)-2-(4-(3,4,5-trimethoxyphenyl) but-3-en-1-yn-1-yl)} benzoate 2d (Yield: $211 \mathrm{mg}, 0.6 \mathrm{mmol}, 60 \%$ )

IR (neat): 3000, 2943, 2839, 2192, 1729, 1578, 1505, 1484, 1465, 1450, $1418,1343,1298,1282,1252,1233,1188,1154,1126,1086,1045,1005$, $951,853,812,758,701,628 \mathrm{~cm}^{-1}$.

${ }^{1} \mathrm{H}$ NMR (300 MHz, $\left.\mathrm{CDCl}_{3}\right) \delta=7.95(\mathrm{~d}, J=7.8 \mathrm{~Hz}, 1 \mathrm{H}), 7.57(\mathrm{~d}, J=7.8 \mathrm{~Hz}$, $1 \mathrm{H}), 7.46(\mathrm{td}, J=7.5 \mathrm{~Hz}, J=1.0 \mathrm{~Hz}, 1 \mathrm{H}), 7.35(\mathrm{dd}, \mathrm{J}=7.5 \mathrm{~Hz}, J=1.0 \mathrm{~Hz}, 1 \mathrm{H}$ ), 7.01 (d, $J=16.2 \mathrm{~Hz}, 1 \mathrm{H}), 6.66(\mathrm{~s}, 2 \mathrm{H}), 6.37$ (d, $J=16.2 \mathrm{~Hz}, 1 \mathrm{H}), 3.95$ (s, 3H), $3.87(\mathrm{~s}, 6 \mathrm{H}), 3.86(\mathrm{~s}, 3 \mathrm{H})$.

${ }^{13} \mathrm{C}$ NMR (75 MHz, $\left.\mathrm{CDCl}_{3}\right) \delta=166.5(\mathrm{Cq}), 153.4(2 \mathrm{Cq}), 141.8(\mathrm{CH}), 138.9$ $(\mathrm{Cq}), 134.0(\mathrm{CH}), 132.0(\mathrm{Cq}), 131.7(\mathrm{CH}), 131.4(\mathrm{Cq}), 130.4(\mathrm{CH}), 127.7$ 
(CH), $124.0(\mathrm{Cq}), 107.7(\mathrm{CH}), 103.6(2 \mathrm{CH}), 93.9(\mathrm{Cq}), 90.5(\mathrm{Cq}), 60.9\left(\mathrm{CH}_{3}\right)$, $56.1\left(2 \mathrm{CH}_{3}\right), 52.2\left(\mathrm{CH}_{3}\right)$.

HRMS Calcd for $\mathrm{C}_{21} \mathrm{H}_{21} \mathrm{O}_{5}[\mathrm{M}+\mathrm{H}]^{+}$353.1389, found 353.1385 .

Methyl (E)-2-(4-(2,5-dimethylyphenyl) but-3-en-1-yn-1-yl) benzoate 2e (Yield: $154 \mathrm{mg}, 0.53 \mathrm{mmol}, 53 \%)$

IR (neat): 2949, 2194, 1730, 1712, 1592, 1564, 1482, 1447, 1432, 1292, $1249,1190,1163,1132,1083,1045,952,882,810,756,699,660 \mathrm{~cm}^{-1}$.

${ }^{1} \mathrm{H} \mathrm{NMR}\left(300 \mathrm{MHz}, \mathrm{CDCl}_{3}\right) \delta=7.97(\mathrm{dd}, J=7.5 \mathrm{~Hz}, J=0.6 \mathrm{~Hz}, 1 \mathrm{H}), 7.59(\mathrm{dd}$, $J=7.5 \mathrm{~Hz}, J=0.6 \mathrm{~Hz}, 1 \mathrm{H}), 7.48(\mathrm{td}, J=7.5 \mathrm{~Hz}, J=1.2 \mathrm{~Hz}, 1 \mathrm{H}), 7.39-7.29(\mathrm{~m}$, $3 \mathrm{H}), 7.05(\mathrm{~m}, 2 \mathrm{H}), 6.37$ (d, $J=16.2 \mathrm{~Hz}, 1 \mathrm{H}), 3.97$ (s, 3H), 2.37 (s, 3H), 2.33 $(\mathrm{s}, 3 \mathrm{H})$.

${ }^{13} \mathrm{C}$ NMR $\left(75 \mathrm{MHz}, \mathrm{CDCl}_{3}\right) \delta=166.7(\mathrm{Cq}), 139.9(\mathrm{CH}), 135.6(\mathrm{Cq}), 135.0$ (Cq), 134.0 (CH), 132.9 (Cq), $131.7(\mathrm{CH}), 130.5$ (CH), $130.4(\mathrm{CH}), 129.5$ (CH), $127.7(\mathrm{CH}), 125.6(\mathrm{CH}), 124.0(\mathrm{Cq}), 108.9(\mathrm{CH}), 94.4(\mathrm{Cq}), 90.0(\mathrm{Cq})$, $52.2\left(\mathrm{CH}_{3}\right), 21.0\left(\mathrm{CH}_{3}\right), 19.3\left(\mathrm{CH}_{3}\right)$, one $\mathrm{C}$ not seen.

HRMS Calcd for $\mathrm{C}_{20} \mathrm{H}_{19} \mathrm{O}_{2}[\mathrm{M}+\mathrm{H}]^{+} 291.1385$, found 291.1386.

Methyl (E)-2-(4-(4-thiomethylphenyl)but-3-en-1-yn-1-yl)benzoate 2f (Yield: $154 \mathrm{mg}, 0.5 \mathrm{mmol}, 50 \%$ )

IR (neat): 3028, 2949, 2192, 1729, 1709, 1670, 1590, 1564, 1482, 1432, $1405,1293,1249,1188,1163,1134,1083,1045,1014,954,852,803,756$, $699,655 \mathrm{~cm}^{-1}$.

${ }^{1} \mathrm{H}$ NMR $\left(300 \mathrm{MHz}, \mathrm{CDCl}_{3}\right) \delta=7.96(\mathrm{dd}, J=7.8 \mathrm{~Hz}, J=1.0 \mathrm{~Hz}, 1 \mathrm{H}), 7.58(\mathrm{dd}$, $J=7.8 \mathrm{~Hz}, J=1.0 \mathrm{~Hz}, 1 \mathrm{H}$ ), 7.47 (td, $J=7.5 \mathrm{~Hz}, J=1.0 \mathrm{~Hz}, 1 \mathrm{H}$ ), $7.38-7.32$ $(\mathrm{m}, 3 \mathrm{H}), 7.21(\mathrm{~d}, J=8.4 \mathrm{~Hz}, 2 \mathrm{H}), 7.04(\mathrm{~d}, J=16.2 \mathrm{~Hz}, 1 \mathrm{H}), 6.41(\mathrm{~d}, J=16.2$ $\mathrm{Hz}, 1 \mathrm{H}), 3.95$ (s, 3H), 2.49 (s, 3H).

${ }^{13} \mathrm{C}$ NMR $\left(75 \mathrm{MHz}, \mathrm{CDCl}_{3}\right) \delta=166.6(\mathrm{Cq}), 141.3(\mathrm{CH}), 139.6(\mathrm{Cq}), 134.0$ (CH), $133.1(\mathrm{Cq}), 131.7(\mathrm{CH}), 131.5(\mathrm{Cq}), 130.4(\mathrm{CH}), 127.7(\mathrm{CH}), 126.8$ (2CH), $126.4(2 \mathrm{CH}), 124.1(\mathrm{Cq}), 107.5(\mathrm{CH}), 94.1(\mathrm{Cq}), 90.5(\mathrm{Cq}), 52.2$ $\left(\mathrm{CH}_{3}\right), 15.5\left(\mathrm{CH}_{3}\right)$.

HRMS Calcd for $\mathrm{C}_{19} \mathrm{H}_{17} \mathrm{O}_{2} \mathrm{~S}[\mathrm{M}+\mathrm{H}]^{+}$309.0949, found 309.0945 .

Methyl (E)-2-(4-(naphthalen-2-yl)but-3-en-1-yn-1-yl) benzoate $2 \mathrm{~g}$ (Yield: $165 \mathrm{mg}, 0.53 \mathrm{mmol}, 53 \%$ )

IR (neat): 3058, 2949, 2193, 1728, 1708, 1633, 1591, 1563, 1483, 1432, 1364, 1290, 1273, 1250, 1189, 1162, 1128, 1084, 1046, 952, 910, 862, $811,756,699,659,625 \mathrm{~cm}^{-1}$.

${ }^{1} \mathrm{H}$ NMR $\left(300 \mathrm{MHz}, \mathrm{CDCl}_{3}\right) \delta=7.98(\mathrm{dd}, J=7.8, J=1.0 \mathrm{~Hz}, 1 \mathrm{H}), 7.85-7.79$ (m, 4H), $7.65-7.61(\mathrm{~m}, 2 \mathrm{H}), 7.51-7.46(\mathrm{~m}, 3 \mathrm{H}), 7.37(\mathrm{td}, J=7.8 \mathrm{~Hz}, J=1.0$ $\mathrm{Hz}, 1 \mathrm{H}), 7.27$ (d, $J=16.2 \mathrm{~Hz}, 1 \mathrm{H}), 6.60$ (d, $J=16.2 \mathrm{~Hz}, 1 \mathrm{H}), 3.99$ (s, 3H).

${ }^{13} \mathrm{C}$ NMR (75 MHz, $\left.\mathrm{CDCl}_{3}\right) \delta=166.6(\mathrm{Cq}), 142.1(\mathrm{CH}), 134.1(\mathrm{CH}), 133.8$ (Cq), $133.6(\mathrm{Cq}), 133.5(\mathrm{Cq}), 131.7(\mathrm{CH}), 131.6(\mathrm{Cq}), 130.5(\mathrm{CH}), 128.5$ (CH), $128.3(\mathrm{CH}), 127.8(\mathrm{CH}), 127.7(\mathrm{CH}), 127.2(\mathrm{CH}), 126.5(\mathrm{CH}), 126.4$ (CH), $124.0(\mathrm{Cq}), 122.8(\mathrm{CH}), 108.6(\mathrm{CH}), 94.2(\mathrm{Cq}), 90.9(\mathrm{Cq}), 52.2\left(\mathrm{CH}_{3}\right)$. HRMS Calcd for $\mathrm{C}_{22} \mathrm{H}_{17} \mathrm{O}_{2}[\mathrm{M}+\mathrm{H}]^{+} 313.1229$, found 313.1226 .

Methyl (E)-2-(4-(4-fluorophenyl)but-3-en-1-yn-1-yl)benzoate $2 \mathrm{~h}$ (Yield: $185 \mathrm{mg}, 0.66 \mathrm{mmol}, 66 \%)$

IR (neat): 2951, 2195, 1729, 1712, 1600, 1565, 1509, 1484, 1448, 1433, 1296, 1285, 1252, 1228, 1191, 1159, 1133, 1085, 1045, 955, 935, 852, $815,791,757,699,659 \mathrm{~cm}^{-1}$.

${ }^{1} \mathrm{H} \mathrm{NMR}\left(300 \mathrm{MHz}, \mathrm{CDCl}_{3}\right) \delta 7.98(\mathrm{~d}, J=7.1 \mathrm{~Hz}, 2 \mathrm{H}), 7.61(\mathrm{~d}, J=7.6 \mathrm{~Hz}, 2 \mathrm{H})$, $7.53-7.36(\mathrm{~m}, 4 \mathrm{H}), 7.11-7.02(\mathrm{~m}, 3 \mathrm{H}), 6.39(\mathrm{~d}, J=16.2 \mathrm{~Hz}, 1 \mathrm{H}), 3.98(\mathrm{~s}$, $3 \mathrm{H})$.

${ }^{13} \mathrm{C}$ NMR $\left(75 \mathrm{MHz}, \mathrm{CDCl}_{3}\right) \delta=166.5(\mathrm{Cq}), 163.0(\mathrm{~d}, J=247.5 \mathrm{~Hz}, \mathrm{Cq}), 140.6$ (CH), 134.1 (CH), 132.5 (Cq), 131.7 (CH), 131.5 (Cq), 130.5 (CH), 128.1 (d,
$J=7.5 \mathrm{~Hz}, \mathrm{CH}), 127.8(\mathrm{CH}), 124.0(\mathrm{Cq}), 115.8(\mathrm{~d}, J=21.8 \mathrm{~Hz}, \mathrm{CH}), 108.1$ $(\mathrm{CH}), 93.7(\mathrm{Cq}), 90.4(\mathrm{Cq}), 52.2\left(\mathrm{CH}_{3}\right)$.

${ }^{19} \mathrm{~F}$ NMR $\left(188 \mathrm{MHz}, \mathrm{CDCl}_{3}\right) \delta=-110.42$.

HRMS Calcd for $\mathrm{C}_{18} \mathrm{H}_{14} \mathrm{O}_{2} \mathrm{~F}[\mathrm{M}+\mathrm{H}]+281.0978$, found 281.0975.

Methyl (E)-2-(4-(2-fluorophenyl)but-3-en-1-yn-1-yl)benzoate 2i (Yield: $179 \mathrm{mg}, 0.64 \mathrm{mmol}, 64 \%$ )

IR (cm-1): 2950, 1729, 1711, 1593, 1565, 1481, 1448, 1432, 1286, 1251, $1228,1189,1164,1133,1082,1046,953,885,827,803,751,698,660$.

${ }^{1} \mathrm{H} \mathrm{NMR}\left(300 \mathrm{MHz}, \mathrm{CDCl}_{3}\right) \delta=7.88(\mathrm{~d}, J=8.5 \mathrm{~Hz}, 1 \mathrm{H}), 7.51(\mathrm{~d}, J=7.8 \mathrm{~Hz}$, 1H), $7.44-7.35(\mathrm{~m}, 2 \mathrm{H}), 7.28(\mathrm{td}, J=7.8,1.1 \mathrm{~Hz}, 1 \mathrm{H}), 7.22-7.09(\mathrm{~m}, 2 \mathrm{H})$, $7.07-6.92(\mathrm{~m}, 2 \mathrm{H}), 6.48(\mathrm{~d}, J=16.4 \mathrm{~Hz}, 1 \mathrm{H}), 3.85(\mathrm{~s}, 3 \mathrm{H})$.

${ }^{13} \mathrm{C} \mathrm{NMR}\left(75 \mathrm{MHz}, \mathrm{CDCl}_{3}\right) \delta=166.6(\mathrm{Cq}), 160.3(\mathrm{~d}, J=250.5 \mathrm{~Hz}, \mathrm{Cq}), 134.5$ (d, $J=2.3 \mathrm{~Hz}, \mathrm{CH}), 134.1(\mathrm{CH}), 131.7(\mathrm{CH}), 131.6(\mathrm{Cq}), 130.5(\mathrm{CH}), 130.0$ (d, $J=8.3 \mathrm{~Hz}, \mathrm{CH}), 127.9(\mathrm{CH}), 127.2(\mathrm{~d}, J=3.0 \mathrm{~Hz}, \mathrm{CH}), 124.3(\mathrm{~d}, J=3.0 \mathrm{~Hz}$, CH), $124.2(\mathrm{Cq}), 123.8(\mathrm{Cq}), 116.0(\mathrm{~d}, J=22.5 \mathrm{~Hz}, \mathrm{CH}), 110.9(\mathrm{~d}, J=6.8 \mathrm{~Hz}$, $\mathrm{CH}), 93.9(\mathrm{Cq}), 91.1(\mathrm{Cq}), 52.2\left(\mathrm{CH}_{3}\right)$.

${ }^{19} \mathrm{~F}$ NMR $\left(188 \mathrm{MHz}, \mathrm{CDCl}_{3}\right) \delta=-114.74$.

HRMS Calcd for $\mathrm{C}_{18} \mathrm{H}_{14} \mathrm{O}_{2} \mathrm{~F}[\mathrm{M}+\mathrm{H}]+281.0978$, found 281.0974.

Methyl (E)-2-(4-(4-trifluoromethylphenyl)but-3-en-1-yn-1-yl) benzoate $2 \mathbf{j}$ (Yield: $132 \mathrm{mg}, 0.4 \mathrm{mmol}, 40 \%$ )

IR (neat): 1729, 1711, 1612, 1592, 1564, 1484, 1433, 1412, 1322, 1289 , $1250,1189,1163,1123,1108,1084,1066,1045,1014,962,945,861$, $815,755,699,664,645 \mathrm{~cm}^{-1}$.

${ }^{1} \mathrm{H} \mathrm{NMR}\left(300 \mathrm{MHz}, \mathrm{CDCl}_{3}\right) \delta=7.98(\mathrm{~d}, J=7.8 \mathrm{~Hz}, 1 \mathrm{H}), 7.61-7.46(\mathrm{~m}, 6 \mathrm{H})$, $7.38(\mathrm{t}, J=7.5 \mathrm{~Hz}, 1 \mathrm{H}), 7.10(\mathrm{~d}, J=16.2 \mathrm{~Hz}, 1 \mathrm{H}), 6.55(\mathrm{~d}, J=16.2 \mathrm{~Hz}, 1 \mathrm{H})$, $3.96(\mathrm{~s}, 3 \mathrm{H})$.

${ }^{13} \mathrm{C}$ NMR $\left(75 \mathrm{MHz}, \mathrm{CDCl}_{3}\right) \delta=166.4(\mathrm{Cq}), 140.1(\mathrm{CH}), 139.6(\mathrm{Cq}), 134.1$ (CH), $131.8(\mathrm{CH}), 131.6(\mathrm{Cq}), 130.5(\mathrm{CH}), 130.1(\mathrm{Cq}), 129.7(\mathrm{CH}), 128.1$ (CH), $126.5(2 \mathrm{CH}), 125.7(2 \mathrm{CH}), 123.7(\mathrm{Cq}), 122.2(\mathrm{Cq}), 111.1(\mathrm{CH}), 93.2$ (Cq), $91.80(\mathrm{Cq}) 52.2\left(\mathrm{CH}_{3}\right)$.

${ }^{19} \mathrm{~F}$ NMR $\left(188 \mathrm{MHz}, \mathrm{CDCl}_{3}\right) \delta=-63.04$.

HRMS Calcd for $\mathrm{C}_{19} \mathrm{H}_{14} \mathrm{O}_{2} \mathrm{~F}_{3}[\mathrm{M}+\mathrm{H}]^{+}$331.0946, found 331.0953.

Methyl (E)-2-(4-(3-nitrophenyl)but-3-en-1-yn-1-yl)benzoate 2k (Yield: $166 \mathrm{mg}, 0.54 \mathrm{mmol}, 54 \%)$

IR (neat): 2951, 1729, 1711, 1565, 1528, 1485, 1433, 1351, 1293, 1254, $1190,1135,1086,1046,952,824,803,758,734,699,674 \mathrm{~cm}^{-1}$.

${ }^{1} \mathrm{H} \mathrm{NMR}\left(300 \mathrm{MHz}, \mathrm{CDCl}_{3}\right) \delta=8.28(\mathrm{~s}, 1 \mathrm{H}), 8.13(\mathrm{~d}, J=7.8 \mathrm{~Hz}, 1 \mathrm{H}), 7.98(\mathrm{~d}$, $J=7.8 \mathrm{~Hz}, 1 \mathrm{H}), 7.73(\mathrm{~d}, J=7.8 \mathrm{~Hz}, 1 \mathrm{H}), 7.61(\mathrm{~d}, J=7.5 \mathrm{~Hz}, 1 \mathrm{H}), 7.55-7.47$ $(\mathrm{m}, 2 \mathrm{H}), 7.40(\mathrm{t}, J=7.2 \mathrm{~Hz}, 1 \mathrm{H}), 7.12(\mathrm{~d}, J=16.2 \mathrm{~Hz}, 1 \mathrm{H}), 6.60(\mathrm{~d}, J=16.2$ $\mathrm{Hz}, 1 \mathrm{H}), 3.96$ (s, 3H).

${ }^{13} \mathrm{C}$ NMR (75 MHz, $\left.\mathrm{CDCl}_{3}\right) \delta=164.3(\mathrm{Cq}), 148.7(\mathrm{Cq}), 139.0(\mathrm{CH}), 138.0$ (2Cq), $134.2(\mathrm{CH}), 131.9(\mathrm{CH}), 131.8(\mathrm{CH}), 130.5(\mathrm{CH}), 129.7(\mathrm{CH}), 128.2$ (CH), $123.5(\mathrm{Cq}), 123.0(\mathrm{CH}), 120.9(\mathrm{CH}), 111.8(\mathrm{CH}), 92.7(\mathrm{Cq}), 92.3(\mathrm{Cq})$, $52.3\left(\mathrm{CH}_{3}\right)$.

HRMS Calcd for $\mathrm{C}_{18} \mathrm{H}_{14} \mathrm{NO}_{4}[\mathrm{M}+\mathrm{H}]^{+}$308.0923, found 308.0922.

Methyl (E)-2-(4-(3,4-dichlorophenyl)but-3-en-1-yn-1-yl)benzoate 21 (Yield: $142 \mathrm{mg}, 0.43 \mathrm{mmol}, 43 \%$ )

IR (neat): 2008, 1729, 1592, 1565, 1473, 1432, 1389, 1294, 1275, 1252, 1190, 1133, 1085, 1046, 1029, 945, 880, 809, 756, 698, 673, $656 \mathrm{~cm}^{-1}$

${ }^{1} \mathrm{H}$ NMR $\left(300 \mathrm{MHz}, \mathrm{CDCl}_{3}\right) \delta=7.99(\mathrm{~d}, J=7.8 \mathrm{~Hz}, 1 \mathrm{H}), 7.61(\mathrm{~d}, J=7.5 \mathrm{~Hz}$, 1H), $7.52-7.47(\mathrm{~m}, 2 \mathrm{H}), 7.44-7.37(\mathrm{~m}, 2 \mathrm{H}), 7.27(\mathrm{dd}, J=8.1, \mathrm{~J}=2.1 \mathrm{~Hz}$, $1 \mathrm{H}), 6.99(\mathrm{~d}, J=16.2 \mathrm{~Hz}, 1 \mathrm{H}), 6.46(\mathrm{~d}, J=16.2 \mathrm{~Hz}, 1 \mathrm{H}), 3.97(\mathrm{~s}, 3 \mathrm{H})$. 
${ }^{13} \mathrm{C}$ NMR $\left(75 \mathrm{MHz}, \mathrm{CDCl}_{3}\right) \delta=166.4(\mathrm{Cq}), 139.1(\mathrm{CH}), 136.4(\mathrm{Cq}), 134.1$ (CH), $133.0(\mathrm{Cq}), 132.4(\mathrm{Cq}), 131.8(\mathrm{CH}), 131.6(\mathrm{Cq}), 130.7(\mathrm{CH}), 130.5$ (CH), $128.1(\mathrm{CH}), 128.0(\mathrm{CH}), 125.4(\mathrm{CH}), 123.7(\mathrm{Cq}), 110.4(\mathrm{CH}), 93.1$ $(\mathrm{Cq}), 91.8(\mathrm{Cq}), 52.2\left(\mathrm{CH}_{3}\right)$.

HRMS Calcd for $\mathrm{C}_{18} \mathrm{H}_{13} \mathrm{O}_{2} \mathrm{Cl}_{2}[\mathrm{M}+\mathrm{H}]^{+} 331.0293$, found 331.0294 .

Methyl (E)-2-(4-(4-acetylphenyl)but-3-en-1-yn-1-yl)benzoate $2 \mathrm{~m}$ (Yield: $195 \mathrm{mg}, 0.64 \mathrm{mmol}, 64 \%)$

IR (neat): 1729, 1712, 1679, 1599, 1564, 1484, 1432, 1410, 1358, 1290, $1269,1250,1183,1134,1085,1046,1015,959,861,814,758,699 \mathrm{~cm}^{-1}$.

${ }^{1} \mathrm{H}$ NMR $\left(300 \mathrm{MHz}, \mathrm{CDCl}_{3}\right) \delta=7.98-7.91(\mathrm{~m}, 3 \mathrm{H}), 7.59(\mathrm{~d}, J=7.5 \mathrm{~Hz}, 1 \mathrm{H})$, $7.51-7.48(\mathrm{~m}, 3 \mathrm{H}), 7.37(\mathrm{t}, J=7.5 \mathrm{~Hz}, 1 \mathrm{H}), 7.10(\mathrm{~d}, J=16.2 \mathrm{~Hz}, 1 \mathrm{H}), 6.57$ (d, $J=16.2 \mathrm{~Hz}, 1 \mathrm{H}), 3.95(\mathrm{~s}, 3 \mathrm{H}), 2.59(\mathrm{~s}, 3 \mathrm{H})$.

${ }^{13} \mathrm{C}$ NMR $\left(75 \mathrm{MHz}, \mathrm{CDCl}_{3}\right) \delta=197.3(\mathrm{Cq}), 166.4(\mathrm{Cq}), 140.7(\mathrm{Cq}), 140.5$ (CH), $136.8(\mathrm{Cq}), 134.1(\mathrm{CH}), 131.8(\mathrm{CH}), 131.6(\mathrm{Cq}), 130.5(\mathrm{CH}), 128.8$ (2CH), $128.1(\mathrm{CH}), 126.4(2 \mathrm{CH}), 123.7(\mathrm{Cq}), 111.2(\mathrm{CH}), 93.4(\mathrm{Cq}), 92.1$ (Cq), $52.2\left(\mathrm{CH}_{3}\right), 26.6\left(\mathrm{CH}_{3}\right)$.

HRMS Calcd for $\mathrm{C}_{20} \mathrm{H}_{17} \mathrm{O}_{3}[\mathrm{M}+\mathrm{H}]^{+}$305.1178, found 305.1171 .

Methyl (E)-2-(4-(4-cyanophenyl)but-3-en-1-yn-1-yl)benzoate 2n (Yield: $164 \mathrm{mg}, 0.57 \mathrm{mmol}, 57 \%$ )

IR (neat): 2951, 2226, 1728, 1709, 1601, 1565, 1484, 1448, 1432, 1412, 1296, 1251, 1191, 1133, 1085, 963,945, 863, 815, 757, 699, $658 \mathrm{~cm}^{-1}$.

${ }^{1} \mathrm{H} \mathrm{NMR}\left(300 \mathrm{MHz}, \mathrm{CDCl}_{3}\right) \delta=7.90(\mathrm{~d}, J=7.9 \mathrm{~Hz}, 1 \mathrm{H}), 7.52-7.40(\mathrm{~m}, 6 \mathrm{H})$, 7.31 (td, $J=7.7,1.2 \mathrm{~Hz}, 1 \mathrm{H}$ ), 6.98 (d, $J=16.2 \mathrm{~Hz}, 1 \mathrm{H}$ ), 6.49 (d, $J=16.2 \mathrm{~Hz}$ $1 \mathrm{H}), 3.87(\mathrm{~s}, 3 \mathrm{H})$, an impurity was observed between 7.60 and $7.75 \mathrm{ppm}$.

${ }^{13} \mathrm{C}$ NMR $\left(75 \mathrm{MHz}, \mathrm{CDCl}_{3}\right) \delta=166.3(\mathrm{Cq}), 140.6(\mathrm{Cq}), 139.6(\mathrm{CH}), 134.2$ (CH), $131.8(2 \mathrm{CH}), 131.6(\mathrm{Cq}), 130.5(\mathrm{CH}), 128.3(\mathrm{CH}), 126.8(2 \mathrm{CH}), 123.5$ (Cq), $118.7(\mathrm{Cq}), 112.3(\mathrm{CH}), 111.7(\mathrm{Cq}), 93.0(\mathrm{Cq}), 92.8(\mathrm{Cq}), 52.2\left(\mathrm{CH}_{3}\right)$.

HRMS Calcd for ${ }_{19} \mathrm{H}_{14} \mathrm{O}_{2}[\mathrm{M}+\mathrm{H}]^{+} 288.1025$, found 288.1022 .

Methyl (Z)-2-(4-(4-methoxyphenyl)but-3-en-1-yn-1-yl) benzoate 2a (Yield: $146 \mathrm{mg}, 0.5 \mathrm{mmol}, 50 \%$ )

IR (neat): 2951, 2837, 2182, 1728, 1711, 1605, 1565, 1510, 1484, 1432, 1408, 1326, 1304, 1279, 1248, 1172, 1132, 1083, 1031, 962, 835, 789, $756,699,653 \mathrm{~cm}^{-1}$.

${ }^{1} \mathrm{H} \mathrm{NMR}\left(300 \mathrm{MHz}, \mathrm{CDCl}_{3}\right) \delta=7.98-7.94(\mathrm{~m}, 3 \mathrm{H}), 7.60(\mathrm{~d}, J=7.5 \mathrm{~Hz}, 1 \mathrm{H})$, $7.49(\mathrm{t}, J=7.5 \mathrm{~Hz}, 1 \mathrm{H}), 7.37(\mathrm{t}, J=7.5 \mathrm{~Hz}, 1 \mathrm{H}), 6.92(\mathrm{~d}, J=8.7 \mathrm{~Hz}, 2 \mathrm{H}), 6.67$ (d, $J=12.0 \mathrm{~Hz}, 1 \mathrm{H}), 5.88$ (d, $J=12.0 \mathrm{~Hz}, 1 \mathrm{H}), 3.89$ (s, 3H), 3.83 (s, 3H).

${ }^{13} \mathrm{C}$ NMR (75 MHz, $\mathrm{CDCl}_{3}$ ) $\delta=166.7$ (Cq), 159.9 (Cq), $138.8(\mathrm{CH}), 134.1$ (CH), 131.7 (CH), 131.5 (Cq), 130.5 (3CH), 129.5 (Cq), $127.8(\mathrm{CH}), 124.1$ (Cq), $113.7(2 \mathrm{CH}), 104.9(\mathrm{CH}), 94.1(\mathrm{Cq}), 93.6(\mathrm{Cq}), 55.3\left(\mathrm{CH}_{3}\right), 52.2\left(\mathrm{CH}_{3}\right)$.

HRMS Calcd for $\mathrm{C}_{19} \mathrm{H}_{17} \mathrm{O}_{3}[\mathrm{M}+\mathrm{H}]^{+} 293.1178$, found 293.1179 .

Methyl (Z)-2-(4-(3,4,5-trimethoxyphenyl)but-3-en-1-yn-1-yl) benzoate 2d (Yield: $204 \mathrm{mg}, 0.58 \mathrm{mmol}, 58 \%$ ) accompanied with less than $2 \%$ of $(E)-\mathbf{2 d}$.

IR (neat): 2838, 1728, 1574, 1506, 1483, 1450, 1422, 1404, 1330, 1299, $1279,1251,1238,1188,1126,1083,1043,1006,963,846,758,700,644$ $\mathrm{cm}^{-1}$.

${ }^{1} \mathrm{H} \mathrm{NMR}\left(300 \mathrm{MHz}, \mathrm{CDCl}_{3}\right) \delta=7.97(\mathrm{dd}, J=7.8 \mathrm{~Hz}, J=1.2 \mathrm{~Hz}, 1 \mathrm{H}), 7.57(\mathrm{dd}$, $J=7.8 \mathrm{~Hz}, J=1.2 \mathrm{~Hz}, 1 \mathrm{H}), 7.47(\mathrm{td}, J=7.5 \mathrm{~Hz}, J=1.2 \mathrm{~Hz}, 1 \mathrm{H}), 7.38(\mathrm{td}, J=7.5$ $\mathrm{Hz}, J=1.2 \mathrm{~Hz}, 1 \mathrm{H}), 7.22(\mathrm{~s}, 2 \mathrm{H}), 6.64(\mathrm{~d}, J=12.0 \mathrm{~Hz}, 1 \mathrm{H}), 5.96(\mathrm{~d}, J=12.0$ $\mathrm{Hz}, 1 \mathrm{H}), 3.86$ (s, 6H), $3.80(\mathrm{~s}, 6 \mathrm{H})$.

${ }^{13} \mathrm{C}$ NMR (75 MHz, $\mathrm{CDCl}_{3}$ ) $\delta=166.4(\mathrm{Cq}), 152.9(\mathrm{Cq}), 138.9(\mathrm{CH}), 138.6$ $(\mathrm{Cq}), 133.8(\mathrm{CH}), 132.1(\mathrm{Cq}), 131.8(\mathrm{CH}), 131.5(\mathrm{Cq}), 130.6(\mathrm{CH}), 128.0$
(CH), $123.8(\mathrm{Cq}), 106.7(\mathrm{CH}), 106.2(2 \mathrm{CH}), 94.7(\mathrm{Cq}), 93.1(\mathrm{Cq}), 60.9\left(\mathrm{CH}_{3}\right)$, $56.1\left(2 \mathrm{CH}_{3}\right), 52.1\left(\mathrm{CH}_{3}\right)$, one $\mathrm{C}$ not seen.

HRMS Calcd for $\mathrm{C}_{21} \mathrm{H}_{21} \mathrm{O}_{5}[\mathrm{M}+\mathrm{H}]^{+}$353.1389, found 353.1395.

Methyl (Z)-2-(4-(naphthalen-2-yl)but-3-en-1-yn-1-yl)benzoate

$2 g$ (Yield: $181 \mathrm{mg}, 0.58 \mathrm{mmol}, 58 \%)$

IR (neat): 1728, 1593, 1564, 1504, 1484, 1432, 1294, 1277, 1251, 1190, $1164,1134,1082,1043,964,950,895,861,822,757,737,698 \mathrm{~cm}^{-1}$.

${ }^{1} \mathrm{H} \mathrm{NMR}\left(300 \mathrm{MHz}, \mathrm{CDCl}_{3}\right) \delta=8.44(\mathrm{~s}, 1 \mathrm{H}), 8.20(\mathrm{dd}, J=8.4 \mathrm{~Hz}, J=1.5 \mathrm{~Hz}$, $1 \mathrm{H}), 8.02(\mathrm{~d}, J=7.8 \mathrm{~Hz}, 1 \mathrm{H}), 7.92-7.82(\mathrm{~m}, 3 \mathrm{H}), 7.67(\mathrm{~d}, J=7.8 \mathrm{~Hz}, 1 \mathrm{H})$, $7.54-7.46(\mathrm{~m}, 3 \mathrm{H}), 7.40(\mathrm{t}, J=7.5 \mathrm{~Hz}, 1 \mathrm{H}), 6.90(\mathrm{~d}, J=12.0 \mathrm{~Hz}, 1 \mathrm{H}), 6.11$ (d, $J=12.0 \mathrm{~Hz}, 1 \mathrm{H}), 3.89(\mathrm{~s}, 3 \mathrm{H})$.

${ }^{13} \mathrm{C}$ NMR (75 MHz, $\left.\mathrm{CDCl}_{3}\right) \delta=166.6(\mathrm{Cq}), 139.3(\mathrm{CH}), 134.2(\mathrm{CH}), 134.1$ (Cq), $133.4(\mathrm{Cq}), 133.3(\mathrm{Cq}), 131.8(\mathrm{CH}), 131.5(\mathrm{Cq}), 130.6(\mathrm{CH}), 128.6$ (CH), $128.5(\mathrm{CH}), 128.0(\mathrm{CH}), 127.8(\mathrm{CH}), 127.6(\mathrm{CH}), 126.5(2 \mathrm{CH}), 126.2$ (CH), $124.0(\mathrm{Cq}), 107.8(\mathrm{CH}), 94.9(\mathrm{Cq}), 93.3(\mathrm{Cq}), 52.3\left(\mathrm{CH}_{3}\right)$

HRMS Calcd for $\mathrm{C}_{22} \mathrm{H}_{17} \mathrm{O}_{2}[\mathrm{M}+\mathrm{H}]^{+} 313.1229$, found 313.1231.

General procedure for the synthesis of isocoumarins 1

To a solution of $2(0.5 \mathrm{mmol})$ in EtOH ( $4 \mathrm{~mL}$ ) was added PTSA ( $26 \mathrm{mg}, 0.15$ mmol). The mixture was stirred at $160^{\circ} \mathrm{C}$ under microwave irradiation (for time see for each compounds). After concentration in vacuo, the crude mixture was diluted in EtOAc, washed with water and dried over $\mathrm{Na}_{2} \mathrm{SO}_{4}$ and purified by chromatography on $\mathrm{SiO}_{2}$.

(E)-3-(4-Methoxystyryl)-1H-isochromen-1-one 1a (Yield: 132 mg, 0.48 $\mathrm{mmol}, 95 \%)$

Reaction time 1.5 h, (yellow solid, m.p. $142.8-143.5^{\circ} \mathrm{C}$ )

IR (neat) : 3070, 2957, 2837, 1728, 1720, 1635, 1599, 1574, 1560, 1511, $1481,1456,1441,1421,1363,1330,1300,1264,1247,1223,1175,1159$, $1111,1058,1026,976,959,911,881,863,829,790,755,732,688,641$ $\mathrm{cm}^{-1}$.

${ }^{1} \mathrm{H} \mathrm{NMR}\left(300 \mathrm{MHz}, \mathrm{CDCl}_{3}\right) \delta=8.25(\mathrm{~d}, J=7.8 \mathrm{~Hz}, 1 \mathrm{H}), 7.65(\mathrm{t}, J=7.5 \mathrm{~Hz}$, 1H), $7.46-7.36(\mathrm{~m}, 5 \mathrm{H}), 6.90(\mathrm{~d}, J=8.7 \mathrm{~Hz}, 2 \mathrm{H}), 6.55(\mathrm{~d}, J=15.9 \mathrm{~Hz}, 1 \mathrm{H})$, $6.38(\mathrm{~s}, 1 \mathrm{H}), 3.83(\mathrm{~s}, 3 \mathrm{H})$.

${ }^{13} \mathrm{C}$ NMR $\left(75 \mathrm{MHz}, \mathrm{CDCl}_{3}\right) \delta=162.2(\mathrm{Cq}), 160.3(\mathrm{Cq}), 153.0(\mathrm{Cq}), 137.8$ (Cq), $134.8(\mathrm{CH}), 132.6(\mathrm{CH}), 129.8(\mathrm{CH}), 128.6(2 \mathrm{CH}), 127.7(\mathrm{CH}), 125.6$ (CH), $120.7(\mathrm{Cq}), 117.2(\mathrm{CH}), 114.3(2 \mathrm{CH}), 104.8(\mathrm{CH}), 55.4\left(\mathrm{CH}_{3}\right)$, one C not seen.

HRMS Calcd for $\mathrm{C}_{18} \mathrm{H}_{15} \mathrm{O}_{3}[\mathrm{M}+\mathrm{H}]^{+}$279.1021, found 279.1015.

(E)-1a was also prepared from chloroenyne 5 (see after) and 4(methoxyphenyl)boronic acid according to the following protocol: To a solution of 5 (206 mg; $1 \mathrm{mmol}$ ) in THF (10 mL) were added successively, 4-(methoxyphenyl)boronic acid (608 mg, $4 \mathrm{mmol}$ ), KF (192 mg, 3.3 mmol), $\mathrm{Pd}_{2} \mathrm{dba}_{3} . \mathrm{CHCl}_{3}(52 \mathrm{mg}, 0.05 \mathrm{mmol}), \mathrm{P}(t-\mathrm{Bu})_{3}(20 \mathrm{mg}, 0.1 \mathrm{mmol})$. The mixture was stirred at $65{ }^{\circ} \mathrm{C}$ for $7 \mathrm{~h}$. After concentration, the crude was diluted in $\mathrm{CH}_{2} \mathrm{Cl}_{2}$ and washed with water. After drying on $\mathrm{Na}_{2} \mathrm{SO}_{4}$ and evaporation in vacuo, the crude was purified by chromatography on $\mathrm{SiO}_{2}$ to give (E)-1a (203 mg, $0.73 \mathrm{mmol}, 73 \%$ ).

(E)-3-(2-Methoxystyryl)-1H-isochromen-1-one 1b (Yield: $113 \mathrm{mg}, 0.41$ $\mathrm{mmol}, 81 \%)$

Reaction time 2.5 h, (yellow solid, m.p. $129.4-130.3^{\circ} \mathrm{C}$ ).

IR (neat): 3048, 3350, 3250, 3216, 2361, 2328, 2192, 2169, 2150, 2034, $1895,1483,1245,754,688 \mathrm{~cm}^{-1}$.

${ }^{1} \mathrm{H} \mathrm{NMR}\left(300 \mathrm{MHz}, \mathrm{CDCl}_{3}\right) \delta=8.30(\mathrm{~d}, J=7.8 \mathrm{~Hz}, 1 \mathrm{H}), 7.80-7.66(\mathrm{~m}, 2 \mathrm{H})$, $7.56-7.41(\mathrm{~m}, 3 \mathrm{H}), 7.35-7.26(\mathrm{~m}, 2 \mathrm{H}), 7.02-6.92(\mathrm{~m}, 2 \mathrm{H}), 6.84(\mathrm{~d}, J=$ $16.2 \mathrm{~Hz}, 1 \mathrm{H}), 6.47(\mathrm{~s}, 1 \mathrm{H}), 3.94(\mathrm{~s}, 3 \mathrm{H})$. 
${ }^{13} \mathrm{C}$ NMR (75 MHz, $\left.\mathrm{CDCl}_{3}\right) \delta=162.2(\mathrm{Cq}), 157.8(\mathrm{Cq}), 153.3(\mathrm{Cq}), 137.8$ (Cq), $134.7(\mathrm{CH}), 129.9(\mathrm{CH}), 129.8(\mathrm{CH}), 128.5(\mathrm{CH}), 127.8(2 \mathrm{CH}), 125.7$ (CH), $124.9(\mathrm{Cq}), 120.7(\mathrm{CH}), 120.3(\mathrm{CH}), 111.1(\mathrm{CH}), 105.4(\mathrm{CH}), 55.4$ $\left(\mathrm{CH}_{3}\right)$.

HRMS Calcd for $\mathrm{C}_{18} \mathrm{H}_{15} \mathrm{O}_{3}[\mathrm{M}+\mathrm{H}]^{+} 279.1021$, found 279.1024 .

(E)-3-(3-Methoxystyryl)-1H-isochromen-1-one 1c (Yield: 88 mg, 0.32 $\mathrm{mmol}, 63 \%)$

Reaction time $3 \mathrm{~h}$, (yellow solid, m.p. $115.0-115.5^{\circ} \mathrm{C}$ ).

IR (neat): 2835, 1720, 1635, 1615, 1596, 1577, 1561, 1481, 1454, 1432, 1363, 1328, 1291, 1272, 1244, 1218, 1164, 1056, 1023, 977, 956, 918, $879,847,820,779,756,729,688 \mathrm{~cm}^{-1}$.

${ }^{1} \mathrm{H}$ NMR $\left(300 \mathrm{MHz}, \mathrm{CDCl}_{3}\right) \delta=8.27(\mathrm{~d}, J=7.8 \mathrm{~Hz}, 1 \mathrm{H}), 7.67(\mathrm{td}, J=7.5 \mathrm{~Hz}, J$ $=1.0 \mathrm{~Hz}, 1 \mathrm{H}), 7.48-7.38(\mathrm{~m}, 3 \mathrm{H}), 7.30(\mathrm{~d}, J=8.1 \mathrm{~Hz}, 1 \mathrm{H}), 7.11(\mathrm{~d}, J=7.8$ $\mathrm{Hz}, 1 \mathrm{H}), 7.03(\mathrm{~s}, 1 \mathrm{H}), 6.87(\mathrm{dd}, J=8.1 \mathrm{~Hz}, J=2.4 \mathrm{~Hz}, 1 \mathrm{H}), 6.67(\mathrm{~d}, J=15.9$ $\mathrm{Hz}, 1 \mathrm{H}), 6.45$ (s, 1H), $3.84(\mathrm{~s}, 3 \mathrm{H})$.

${ }^{13} \mathrm{C}$ NMR $\left(75 \mathrm{MHz}, \mathrm{CDCl}_{3}\right) \delta=162.0(\mathrm{Cq}), 160.0(\mathrm{Cq}), 152.5(\mathrm{Cq}), 137.5$ (Cq), $137.2(\mathrm{Cq}), 134.8(\mathrm{CH}), 132.9(\mathrm{CH}), 129.8(2 \mathrm{CH}), 128.1(\mathrm{CH}), 125.8$ (CH), 120.9 (Cq), 119.8 (2CH), $114.6(\mathrm{CH}), 112.3(\mathrm{CH}), 105.8(\mathrm{CH}), 55.3$ $\left(\mathrm{CH}_{3}\right)$.

HRMS Calcd for $\mathrm{C}_{18} \mathrm{H}_{15} \mathrm{O}_{3}[\mathrm{M}+\mathrm{H}]^{+} 279.1021$, found 279.1019.

(E)-3-(3,4,5-Trimethoxystyryl)-1H-isochromen-1-one 1d (Yield: 128 $\mathrm{mg}, 0.38 \mathrm{mmol}, 76 \%$ )

Reaction time $1.5 \mathrm{~h}$, (yellow solid, m.p. $231.7-232.6^{\circ} \mathrm{C}$ ).

IR (neat): 3002, 2938, 1728, 1634, 1616, 1578, 1560, 1504, 1481, 1368, 1342, 1329, 1244, 1220, 1184, 1149, 1123, 1057, 1023, 1005, 969, 890, $844,814,768,732,716,691,663,638 \mathrm{~cm}^{-1}$.

${ }^{1} \mathrm{H} \mathrm{NMR}\left(300 \mathrm{MHz}, \mathrm{CDCl}_{3}\right) \delta=8.27(\mathrm{~d}, J=7.8 \mathrm{~Hz}, 1 \mathrm{H}), 7.67(\mathrm{td}, J=8.1 \mathrm{~Hz}, J$ $=1.0 \mathrm{~Hz}, 1 \mathrm{H}), 7.48-7.34(\mathrm{~m}, 3 \mathrm{H}), 6.74(\mathrm{~s}, 2 \mathrm{H}), 6.60(\mathrm{~d}, J=15.9 \mathrm{~Hz}, 1 \mathrm{H})$, $6.45(\mathrm{~s}, 1 \mathrm{H}), 3.90(\mathrm{~s}, 6 \mathrm{H}), 3.88(\mathrm{~s}, 3 \mathrm{H})$.

${ }^{13} \mathrm{C}$ NMR $\left(75 \mathrm{MHz}, \mathrm{CDCl}_{3}\right) \delta=162.0(\mathrm{Cq}), 153.5(\mathrm{Cq}), 152.6(\mathrm{Cq}), 139.0$ (Cq), $137.6(\mathrm{Cq}), 134.9(\mathrm{CH}), 133.0(\mathrm{CH}), 131.4(\mathrm{Cq}), 129.8(\mathrm{CH}), 128.0$ (CH), $125.8(\mathrm{CH}), 120.8(\mathrm{Cq}), 118.8(\mathrm{CH}), 105.5(\mathrm{CH}), 104.3(2 \mathrm{CH}), 61.0$ $\left(\mathrm{CH}_{3}\right), 56.1\left(2 \mathrm{CH}_{3}\right)$.

HRMS Calcd for $\mathrm{C}_{20} \mathrm{H}_{19} \mathrm{O}_{5}[\mathrm{M}+\mathrm{H}]^{+} 339.1232$, found 339.1227 .

(E)-3-(2,5-Dimethylstyryl)-1H-isochromen-1-one 1e (Yield: $106 \mathrm{mg}$, $0.39 \mathrm{mmol}, 77 \%$ )

Reaction time 2 h, (yellow solid, m.p. $120.3-121.5^{\circ} \mathrm{C}$ ).

IR (neat): 1730, 1635, 1615, 1561, 1482, 1455, 1363, 1329, 1286, 1220, $1162,1057,1025,959,820,755,688 \mathrm{~cm}^{-1}$.

${ }^{1} \mathrm{H}$ NMR $\left(300 \mathrm{MHz}, \mathrm{CDCl}_{3}\right) \delta=8.29(\mathrm{~d}, J=7.8 \mathrm{~Hz}, 1 \mathrm{H}), 7.71-7.62(\mathrm{~m}, 2 \mathrm{H})$, $7.49-7.38(\mathrm{~m}, 3 \mathrm{H}), 7.11-7.38(\mathrm{~m}, 2 \mathrm{H}), 6.61(\mathrm{~d}, J=15.6 \mathrm{~Hz}, 1 \mathrm{H}), 6.46(\mathrm{~s}$, 1H), $2.42(\mathrm{~s}, 3 \mathrm{H}), 2.35(\mathrm{~s}, 3 \mathrm{H})$.

${ }^{13} \mathrm{C}$ NMR (75 MHz, $\mathrm{CDCl}_{3}$ ) $\delta=162.2(\mathrm{Cq}), 152.9(\mathrm{Cq}), 137.6(\mathrm{Cq}), 135.6$ (Cq), $134.8(\mathrm{CH}), 134.6(\mathrm{Cq}), 134.0(\mathrm{Cq}), 130.7(\mathrm{CH}), 130.6(\mathrm{CH}), 129.9$ (CH), $129.5(\mathrm{CH}), 128.0(\mathrm{CH}), 126.0(\mathrm{CH}), 125.7(\mathrm{CH}), 120.9(\mathrm{Cq}), 120.5$ $(\mathrm{CH}), 105.6(\mathrm{CH}), 21.0\left(\mathrm{CH}_{3}\right), 19.5\left(\mathrm{CH}_{3}\right)$.

HRMS Calcd for $\mathrm{C}_{19} \mathrm{H}_{17} \mathrm{O}_{2}[\mathrm{M}+\mathrm{H}]^{+}$277.1229, found 277.1219.

(E)-3-(4-(Methylthio)styryl)-1H-isochromen-1-one 1f (Yield: $132 \mathrm{mg}$, $0.45 \mathrm{mmol}, 90 \%$ )

Reaction time 3 h, (yellow solid, m.p. 148.2 - $148.6^{\circ} \mathrm{C}$ ).

IR (neat): 2920, 1720, 1632, 1615, 1588, 1561, 1480, 1437, 1405, 1363, 1331, 1282, 1224, 1186, 1161, 1093,1057, 1027, 976, 957, 910, 880, 860, $825,805,754,730,688,638 \mathrm{~cm}^{-1}$.
${ }^{1} \mathrm{H}$ NMR (300 MHz, $\left.\mathrm{CDCl}_{3}\right) \delta=8.25(\mathrm{~d}, J=7.8 \mathrm{~Hz}, 1 \mathrm{H}), 7.66(\mathrm{t}, J=7.8 \mathrm{~Hz}$, $1 \mathrm{H}), 7.46-7.34(\mathrm{~m}, 5 \mathrm{H}), 7.22(\mathrm{~d}, J=8.1 \mathrm{~Hz}, 2 \mathrm{H}), 6.62(\mathrm{~d}, J=15.9 \mathrm{~Hz}, 1 \mathrm{H})$, $6.41(\mathrm{~s}, 1 \mathrm{H}), 2.49(\mathrm{~s}, 3 \mathrm{H})$.

${ }^{13} \mathrm{C}$ NMR (75 MHz, $\left.\mathrm{CDCl}_{3}\right) \delta=162.0(\mathrm{Cq}), 152.7(\mathrm{Cq}), 139.8(\mathrm{Cq}), 137.6$ (Cq), $134.8(\mathrm{CH}), 132.5(\mathrm{Cq}), 132.3(\mathrm{CH}), 129.8(\mathrm{CH}), 128.0(\mathrm{CH}), 127.5$ $(2 \mathrm{CH}), 126.4(2 \mathrm{CH}), 125.7(\mathrm{CH}), 120.8(\mathrm{Cq}), 118.6(\mathrm{CH}), 105.5(\mathrm{CH}), 15.5$ $\left(\mathrm{CH}_{3}\right)$.

HRMS Calcd for $\mathrm{C}_{18} \mathrm{H}_{15} \mathrm{O}_{2} \mathrm{~S}[\mathrm{M}+\mathrm{H}]^{+} 295.0793$, found 295.0789.

(E)-3-(2-(Naphthalen-2-yl)vinyl)-1H-isochromen-1-one 1g (Yield: 128 $\mathrm{mg}, 0.43 \mathrm{mmol}, 86 \%$ )

Reaction time 5 h, (yellow solid, m.p. $223.1-223.9^{\circ} \mathrm{C}$ ).

IR (neat): 1740, 1632, 1480, 1364, 1224, 1159, 1054, 1024, 962, 854, 817, $747,686 \mathrm{~cm}^{-1}$.

${ }^{1} \mathrm{H} \mathrm{NMR}\left(300 \mathrm{MHz}, \mathrm{CDCl}_{3}\right) \delta=8.30(\mathrm{~d}, J=8.1 \mathrm{~Hz}, 1 \mathrm{H}), 7.90-7.58(\mathrm{~m}, 4 \mathrm{H})$, $7.71-7.41(\mathrm{~m}, 3 \mathrm{H}), 7.50-7.41(\mathrm{~m}, 4 \mathrm{H}), 6.81(\mathrm{~d}, J=15.9 \mathrm{~Hz}, 2 \mathrm{H}), 6.49$ (s, $2 \mathrm{H})$.

${ }^{13} \mathrm{C}$ NMR (75 MHz, $\mathrm{CDCl}_{3}$ ) $\delta=165.0(\mathrm{Cq}), 162.1(\mathrm{Cq}), 152.7(\mathrm{Cq}), 137.6$ $(\mathrm{Cq}), 134.8(\mathrm{CH}), 133.6(\mathrm{Cq}), 133.3(\mathrm{Cq}), 133.0(\mathrm{CH}), 129.9(\mathrm{CH}), 128.5$ $(\mathrm{CH}), 128.3(\mathrm{CH}), 128.2(\mathrm{CH}), 128.2(\mathrm{CH}), 127.7(\mathrm{CH}), 126.6(2 \mathrm{CH}), 125.8$ (CH), $123.3(\mathrm{CH}), 120.9(\mathrm{Cq}), 119.7(\mathrm{CH}), 105.8(\mathrm{CH})$.

HRMS Calcd for $\mathrm{C}_{21} \mathrm{H}_{15} \mathrm{O}_{2}[\mathrm{M}+\mathrm{H}]+299.1072$, found 299.1074 .

(E)-3-(4-Fluorostyryl)-1H-isochromen-1-one 1h (Yield: 126 mg, 0.48 mmol, 95\%)

Reaction time 5 h, (yellow solid, m.p. $164.4-165.1^{\circ} \mathrm{C}$ ).

IR (neat): 1728, 1723, 1639, 1618, 1599, 1562, 1509, 1482, 1415, 1366, $1329,1293,1234,1222,1160,1098,1058,1027,977,959,832,801,755$, $689 \mathrm{~cm}^{-1}$.

${ }^{1} \mathrm{H}$ NMR $\left(300 \mathrm{MHz}, \mathrm{CDCl}_{3}\right) \delta=8.27(\mathrm{~d}, J=7.5 \mathrm{~Hz}, 1 \mathrm{H}), 7.71-7.65(\mathrm{~m}, 1 \mathrm{H})$, $7.50-7.37(\mathrm{~m}, 5 \mathrm{H}), 7.06(\mathrm{t}, J=8.7 \mathrm{~Hz}, 2 \mathrm{H}), 6.60(\mathrm{~d}, J=15.9 \mathrm{~Hz}, 1 \mathrm{H}), 6.44$ (s, $1 \mathrm{H})$.

${ }^{13} \mathrm{C}$ NMR (75 MHz, $\mathrm{CDCl}_{3}$ ) $\delta=163.0(\mathrm{~d}, J=247.9 \mathrm{~Hz}, \mathrm{Cq}), 162.0(\mathrm{Cq}), 152.4$ (Cq), $137.5(\mathrm{Cq}), 134.9(\mathrm{CH}), 132.1(\mathrm{~d}, J=3.1 \mathrm{~Hz}, \mathrm{Cq}), 131.7(\mathrm{CH}), 129.8$ $(\mathrm{CH}), 128.8(\mathrm{~d}, J=8.1 \mathrm{~Hz}, \mathrm{CH}), 128.1(\mathrm{CH}), 125.8(\mathrm{CH}), 120.8(\mathrm{Cq}), 119.2$ $(\mathrm{CH}), 115.9(\mathrm{~d}, J=21.7 \mathrm{~Hz}, \mathrm{CH}), 105.8(\mathrm{CH})$.

${ }^{19} \mathrm{~F}$ NMR $\left(188 \mathrm{MHz}, \mathrm{CDCl}_{3}\right) \delta=-110.06$.

HRMS Calcd for $\mathrm{C}_{17} \mathrm{H}_{12} \mathrm{O}_{2} \mathrm{~F}[\mathrm{M}+\mathrm{H}]+267.0821$, found 267.0824.

(E)-3-(2-Fluorostyryl)-1H-isochromen-1-one 1i (Yield: $91 \mathrm{mg}, 0.34$ mmol, 67\%)

Reaction time 5 h, (yellow solid, m.p. 164.9 - $165.5^{\circ} \mathrm{C}$ ).

IR (neat): 3134, 2334, 2202, 2157, 1995, 1725, 1707, $1496 \mathrm{~cm}^{-1}$.

${ }^{1} \mathrm{H}$ NMR (300 MHz, $\left.\mathrm{CDCl}_{3}\right) \delta=8.31(\mathrm{~d}, J=8.1 \mathrm{~Hz}, 1 \mathrm{H}), 7.68-7.40(\mathrm{~m}, 1 \mathrm{H})$, 7.43-7.56 (m, $4 \mathrm{H}), 7.26-7.31(\mathrm{~m}, 2 \mathrm{H}), 7.07-7.20(\mathrm{~m}, 2 \mathrm{H}), 6.86(\mathrm{dd}, J=16.2$, $\mathrm{J}=1.6 \mathrm{~Hz}, 1 \mathrm{H}), 6.51(\mathrm{~s}, 1 \mathrm{H})$.

${ }^{13} \mathrm{C}$ NMR (101 MHz, $\left.\mathrm{CDCl}_{3}\right) \delta=162.0(\mathrm{Cq}), 161.1(\mathrm{~d}, \mathrm{~J}=253.1 \mathrm{~Hz}, \mathrm{Cq}), 152.6$ $(\mathrm{Cq}), 137.5(\mathrm{Cq}), 134.9(\mathrm{CH}), 130.1(\mathrm{~d}, \mathrm{~J}=8.5 \mathrm{~Hz}, \mathrm{CH}), 130.0(\mathrm{CH}), 128.8(\mathrm{~d}$, $\mathrm{J}=2.9 \mathrm{~Hz}, \mathrm{CH}), 128.3(\mathrm{CH}), 126.0(\mathrm{~d}, \mathrm{~J}=6.7 \mathrm{~Hz}, \mathrm{CH}), 124.5(\mathrm{~d}, \mathrm{~J}=3.1 \mathrm{~Hz}$, $\mathrm{CH}), 124.0(\mathrm{Cq}), 123.9(\mathrm{Cq}), 122.4(\mathrm{~d}, \mathrm{~J}=8.1 \mathrm{~Hz}, \mathrm{CH}), 121.1(\mathrm{Cq}), 116.2(\mathrm{~d}$, $\mathrm{J}=22.1 \mathrm{~Hz}, \mathrm{CH}), 106.5(\mathrm{CH})$.

${ }^{19} \mathrm{~F}$ NMR (188 MHz, $\left.\mathrm{CDCl}_{3}\right) \delta=-115.4$.

HRMS Calcd for $\mathrm{C}_{17} \mathrm{H}_{12} \mathrm{FO}_{2}[\mathrm{M}+\mathrm{H}]^{+}$267.0821, found 267.0821.

(E)-3-(4-(Trifluoromethyl)styryl)-1H-isochromen-1-one 1j (Yield: 156 $\mathrm{mg}, 0.49 \mathrm{mmol}, 99 \%)$ 
Reaction time 5 h, (yellow solid, m. p. 181.7 - $182.5^{\circ} \mathrm{C}$ ).

IR (neat): 1783, 1729, 1618, 1482, 1414, 1371, 1324, 1260, 1163, 1107, $1067,1015,967,872,837,754,686,638 \mathrm{~cm}^{-1}$.

${ }^{1} \mathrm{H}$ NMR $\left(300 \mathrm{MHz}, \mathrm{CDCl}_{3}\right) \delta=8.29(\mathrm{~d}, J=7.8 \mathrm{~Hz}, 1 \mathrm{H}), 7.70(\mathrm{td}, J=7.8 \mathrm{~Hz}, J$ $=1.0 \mathrm{~Hz}, 1 \mathrm{H}), 7.64-7.57(\mathrm{~m}, 4 \mathrm{H}), 7.52-7.42(\mathrm{~m}, 3 \mathrm{H}), 6.76(\mathrm{~d}, J=16.2 \mathrm{~Hz}$, $1 \mathrm{H}), 6.52(\mathrm{~s}, 1 \mathrm{H})$.

${ }^{13} \mathrm{C}$ NMR (75 MHz, $\mathrm{CDCl}_{3}$ ) $\delta=166.1(\mathrm{Cq}), 161.8(\mathrm{Cq}), 151.9(\mathrm{Cq}), 139.3$ $(\mathrm{Cq}), 137.2(\mathrm{Cq}), 134.9(\mathrm{CH}), 131.2(\mathrm{CH}), 129.9(\mathrm{CH}), 128.5(\mathrm{CH}), 127.2$ $(2 \mathrm{CH}), 126.0(\mathrm{CH}), 125.8(2 \mathrm{CH}), 121.8(\mathrm{CH}), 121.1(\mathrm{Cq}), 106.9(\mathrm{CH})$.

${ }^{19} \mathrm{~F}$ NMR $\left(188 \mathrm{MHz}, \mathrm{CDCl}_{3}\right) \delta=-63.01$.

HRMS Calcd for $\mathrm{C}_{19} \mathrm{H}_{14} \mathrm{O}_{2} \mathrm{~F}_{3}[\mathrm{M}+\mathrm{H}]+317.0789$, found 317.0793.

(E)-3-(3-Nitrostyryl)-1H-isochromen-1-one 1k (Yield: $62 \mathrm{mg}, 0.21$ mmol, 41\%)

Reaction time 6 h, (yellow solid, m.p. 248.0 - $249.0^{\circ} \mathrm{C}$ ).

IR (neat):.1782, 1737, 1620, 1563, 1528, 1482, 1352, 1327, 1225, 1169, $1056,1026,964,833,803,755,735,686,674 \mathrm{~cm}^{-1}$.

${ }^{1} \mathrm{H}$ NMR (400 MHz, DMSO) $\delta=8.48(\mathrm{~s}, 1 \mathrm{H}), 8.18-8.07(\mathrm{~m}, 3 \mathrm{H}), 7.83(\mathrm{t}, J=$ $7.6 \mathrm{~Hz}, 1 \mathrm{H}), 7.71-7.64(\mathrm{~m}, 2 \mathrm{H}), 7.59(\mathrm{t}, J=7.6 \mathrm{~Hz}, 1 \mathrm{H}), 7.42(\mathrm{~d}, J=16.0 \mathrm{~Hz}$, $1 \mathrm{H}), 7.25(\mathrm{~d}, J=16.0 \mathrm{~Hz}, 1 \mathrm{H}), 6.94(\mathrm{~s}, 1 \mathrm{H})$.

${ }^{13} \mathrm{C}$ NMR (101 MHz, DMSO) $\delta=170.9$ (Cq), $160.4(\mathrm{Cq}), 151.2(\mathrm{Cq}), 137.4$ $(\mathrm{Cq}), 136.5(\mathrm{Cq}), 134.8(\mathrm{CH}), 132.8(\mathrm{CH}), 129.8(\mathrm{CH}), 128.9(\mathrm{CH}), 128.6$ $(\mathrm{CH}), 128 .(\mathrm{CH}), 126.1(\mathrm{CH}), 122.9(\mathrm{CH}), 122.4(\mathrm{CH}), 120.8(\mathrm{CH}), 120.1$ (Cq), $106.8(\mathrm{CH})$, one $\mathrm{C}$ not seen.

HRMS Calcd for $\mathrm{C}_{18} \mathrm{H}_{14} \mathrm{NO}_{4}[\mathrm{M}+\mathrm{H}]^{+}$308.0923, found 308.0922.

(E)-3-(3,4-dichlorostyryl)-1H-isochromen-1-one 11 (Yield: 106 mg, 0.34 mmol, 67\%)

Reaction time 5 h, (yellow solid, m.p. $223.3-224.2^{\circ} \mathrm{C}$ ).

IR (neat):.1721, 1617, 1477, 1369, 1226, 1134, 1064, 1027, 958, 823, 750 $685 \mathrm{~cm}^{-1}$

${ }^{1} \mathrm{H} \mathrm{NMR}\left(300 \mathrm{MHz}, \mathrm{CDCl}_{3}\right) \delta=7.99(\mathrm{~d}, J=7.8 \mathrm{~Hz}, 1 \mathrm{H}), 7.61(\mathrm{~d}, J=7.5 \mathrm{~Hz}$, $1 \mathrm{H}), 7.52-7.47(\mathrm{~m}, 2 \mathrm{H}), 7.44-7.37(\mathrm{~m}, 2 \mathrm{H}), 7.27(\mathrm{dd}, J=8.1, \mathrm{~J}=2.1 \mathrm{~Hz}$ 1H), $6.99(\mathrm{~d}, J=16.2 \mathrm{~Hz}, 1 \mathrm{H}), 6.46(\mathrm{~d}, J=16.2 \mathrm{~Hz}, 1 \mathrm{H}), 3.97(\mathrm{~s}, 3 \mathrm{H})$.

${ }^{13} \mathrm{C}$ NMR (75 MHz, $\mathrm{CDCl}_{3}$ ) $\delta=166.4(\mathrm{Cq}), 139.1(\mathrm{CH}), 136.4(\mathrm{Cq}), 134.1$ $(\mathrm{CH}), 133.0(\mathrm{Cq}), 132.4(\mathrm{Cq}), 131.8(\mathrm{CH}), 131.6(\mathrm{Cq}), 130.7(\mathrm{CH}), 130.5$ (CH), $128.1(\mathrm{CH}), 128.0(\mathrm{CH}), 125.4(\mathrm{CH}), 123.7(\mathrm{Cq}), 110.4(\mathrm{CH}), 93.1$ (Cq), $91.8(\mathrm{Cq}), 52.2\left(\mathrm{CH}_{3}\right)$.

HRMS Calcd for $\mathrm{C}_{17} \mathrm{H}_{11} \mathrm{O}_{2} \mathrm{Cl}_{2}[\mathrm{M}+\mathrm{H}]^{+}$317.0136, found 317.0134 .

(E)-3-(4-acetylstyryl)-1H-isochromen-1-one 1m (Yield: $44 \mathrm{mg}, 0.15$ mmol, 30\%)

Reaction time 3.5 h, (yellow solid, m. p. 225.9 - $227.3^{\circ} \mathrm{C}$ ).

IR (neat): 1723, 1673, 1616, 1598, 1563, 1483, 1410, 1357, 1298, 1268, $1181,1065,1027,958,837,812,768,718,690,636 \mathrm{~cm}^{-1}$

${ }^{1} \mathrm{H}$ NMR (300 MHz, $\left.\mathrm{CDCl}_{3}\right) \delta=8.29(\mathrm{~d}, J=8.1 \mathrm{~Hz}, 1 \mathrm{H}), 7.96(\mathrm{~d}, J=8.2 \mathrm{~Hz}$, $2 \mathrm{H}), 7.70(\mathrm{t}, J=7.5 \mathrm{~Hz}, 1 \mathrm{H}), 7.59(\mathrm{~d}, J=8.2 \mathrm{~Hz}, 2 \mathrm{H}), 7.52-7.43(\mathrm{~m}, 3 \mathrm{H})$, $6.80(\mathrm{~d}, J=15.9 \mathrm{~Hz}, 1 \mathrm{H}), 6.53(\mathrm{~s}, 1 \mathrm{H}), 2.61(\mathrm{~s}, 3 \mathrm{H})$.

${ }^{13} \mathrm{C}$ NMR (75 MHz, $\mathrm{CDCl}_{3}$ ) $\delta=197.3(\mathrm{Cq}), 161.8(\mathrm{Cq}), 152.0(\mathrm{Cq}), 140.3$ $(\mathrm{Cq}), 137.2(\mathrm{Cq}), 136.8(\mathrm{Cq}), 134.9(\mathrm{CH}), 131.5(\mathrm{CH}), 129.9(\mathrm{CH}), 128.9$ (2CH), $128.5(\mathrm{CH}), 127.2(\mathrm{CH}), 126.0(\mathrm{CH}), 121.9(\mathrm{CH}), 121.1(\mathrm{Cq}), 107.0$ (CH), $26.6\left(\mathrm{CH}_{3}\right)$.

HRMS Calcd for $\mathrm{C}_{19} \mathrm{H}_{15} \mathrm{O}_{3}[\mathrm{M}+\mathrm{H}]^{+}$291.1021, found 291.1017 .

(E)-4-(2-(1-oxo-1H-isochromen-3-yl)vinyl)benzonitrile 1n (Yield: 27 $\mathrm{mg}, 0.1 \mathrm{mmol}, 20 \%)$
Reaction time 5 h, (yellow solid, m.p. 196.3 - $197.6^{\circ} \mathrm{C}$ ).

IR (neat): 1723, 1655, 1616, 1599, 1481, 1411, 1373, 1327, 1229, 1108, $1061,1023,960,871,837,753,730,686,634 \mathrm{~cm}^{-1}$.

${ }^{1} \mathrm{H}$ NMR $\left(300 \mathrm{MHz}, \mathrm{CDCl}_{3}\right) \delta=8.31(\mathrm{~d}, J=8.1 \mathrm{~Hz}, 1 \mathrm{H}), 7.75-7.41(\mathrm{~m}, 8 \mathrm{H})$, $6.79(\mathrm{~d}, J=15.4 \mathrm{~Hz}, 1 \mathrm{H}), 6.55(\mathrm{~s}, 1 \mathrm{H})$.

${ }^{13} \mathrm{C}$ NMR (75 MHz, $\left.\mathrm{CDCl}_{3}\right) \delta=161.6(\mathrm{Cq}), 151.6(\mathrm{Cq}), 140.2(\mathrm{Cq}), 137.1$ (Cq), $135.0(\mathrm{CH}), 132.6(\mathrm{CH}), 130.7(\mathrm{CH}), 130.0(\mathrm{CH}), 128.7(\mathrm{CH}), 127.4$ $(\mathrm{CH}), 126.1(\mathrm{CH}), 122.8(\mathrm{CH}), 121.3(\mathrm{Cq}), 111.8(\mathrm{Cq}), 107.6(\mathrm{CH}), 105.7$ (Cq).

HRMS Calcd for $\mathrm{C}_{18} \mathrm{H}_{12} \mathrm{NO}_{2}[\mathrm{M}+\mathrm{H}]^{+}$274.0868, found 274.0867.

(E)-3-(2-Chlorovinyl)-1H-isochromen-1-one 5 (Yield: $155 \mathrm{mg}, 0.75$ mmol, 75\%)

To a solution of $(E)-4$ (220 mg; $1 \mathrm{mmol})$ in EtOH ( $4 \mathrm{~mL}$ ) was added $52 \mathrm{mg}$ $(0.3 \mathrm{mmol})$ of PTSA and $53 \mathrm{mg}(0.3 \mathrm{mmol})$ of $\mathrm{PdCl}_{2}$. The solution was stirred for $1 \mathrm{~h}$ under reflux and concentrated. The crude was then dissolved in EtOAc and washed with water. After extraction and drying, the crude was purified by chromatography on $\mathrm{SiO}_{2}$ to give $(E)-\mathbf{5}(155 \mathrm{mg}$, $0.75 \mathrm{mmol}, 75 \%$ ) as a white solid yellow solid, m.p. $91.2-91.4{ }^{\circ} \mathrm{C}$.

IR (neat): 3401, 3385, 3246, 3139, 3100, 2365, 2191, $1966 \mathrm{~cm}^{-1}$.

${ }^{1} \mathrm{H}$ NMR (300 MHz, $\left.\mathrm{CDCl}_{3}\right) \delta=8.26(\mathrm{~d}, J=7.8 \mathrm{~Hz}, 1 \mathrm{H}), 7.69(\mathrm{t}, J=7.5 \mathrm{~Hz}$, $1 \mathrm{H}), 7.49(\mathrm{t}, J=7.5 \mathrm{~Hz}, 1 \mathrm{H}), 7.41(\mathrm{~d}, J=7.8 \mathrm{~Hz}, 1 \mathrm{H}), 7.05(\mathrm{~d}, J=13.2 \mathrm{~Hz}, 1 \mathrm{H})$, $6.46(\mathrm{~d}, J=13.2 \mathrm{~Hz}, 1 \mathrm{H}), 6.34(\mathrm{~s}, 1 \mathrm{H})$.

${ }^{13} \mathrm{C}$ NMR (75 MHz, $\mathrm{CDCl}_{3}$ ) $\delta=161.3(\mathrm{Cq}), 149.9(\mathrm{Cq}), 136.9(\mathrm{Cq}), 135.0$ (CH), $129.9(\mathrm{CH}), 128.6(\mathrm{CH}), 126.0(\mathrm{CH}), 125.1(\mathrm{CH}), 124.7(\mathrm{CH}), 121.0$ (Cq), $105.5(\mathrm{CH})$.

HRMS Calcd for $\mathrm{C}_{11} \mathrm{H}_{7} \mathrm{ClNaO}_{2}[\mathrm{M}+\mathrm{Na}]^{+} 229.0032$, found 229.0041 .

(Z)-3-(2-Chlorovinyl)-1H-isochromen-1-one 5 (Yield: $31 \mathrm{mg}, 0.15 \mathrm{mmol}$, $15 \%)$

To a solution of (Z)-4 (220 mg, $1 \mathrm{mmol})$ in EtOH (4 mL) was added $52 \mathrm{mg}$ $(0.3 \mathrm{mmol})$ of PTSA and $53 \mathrm{mg}(0.3 \mathrm{mmol})$ of $\mathrm{PdCl}_{2}$. The solution was stirred for $1 \mathrm{~h}$ under reflux and concentrated. The crude was then dissolved in EtOAc and washed with water. After extraction and drying, the crude mixture was purified by chromatography to furnish $(Z)-\mathbf{5}$ (31 $\mathrm{mg}, 0.15 \mathrm{mmol}, 15 \%)$ and (E)-5 (43 mg, $0.21 \mathrm{mmol}, 21 \%$ ) which could be separated on $\mathrm{SiO}_{2}$.

$(Z)-5$

IR (neat): 1751, 1728, 1631, 1483, 1288, 1200, 1161, 1139, 1052, 1023, $971,838,791,756,688 \mathrm{~cm}^{-1}$.

${ }^{1} \mathrm{H}$ NMR $\left(300 \mathrm{MHz}, \mathrm{CDCl}_{3}\right) \delta=8.29(\mathrm{~d}, J=8.1 \mathrm{~Hz}, 1 \mathrm{H}), 7.72(\mathrm{~d}, J=7.5 \mathrm{~Hz}$, 1H), $7.55-7.47(\mathrm{~m}, 2 \mathrm{H}), 7.19(\mathrm{~s}, 1 \mathrm{H}), 6.46(\mathrm{~d}, J=8.7 \mathrm{~Hz}, 1 \mathrm{H}), 6.41(\mathrm{~d}, J=$ $8.7 \mathrm{~Hz}, 1 \mathrm{H})$.

${ }^{13} \mathrm{C}$ NMR (75 MHz, $\left.\mathrm{CDCl}_{3}\right) \delta=161.8(\mathrm{Cq}), 149.4(\mathrm{Cq}), 136.8(\mathrm{Cq}), 134.9$ $(\mathrm{CH}), 129.6(\mathrm{CH}), 128.9(\mathrm{CH}), 126.4(\mathrm{CH}), 122.5(\mathrm{CH}), 122.4(\mathrm{CH}), 121.0$ (Cq), $108.1(\mathrm{CH})$.

Calculated for $\mathrm{C}_{11} \mathrm{H}_{8} \mathrm{O}_{2}{ }^{35} \mathrm{Cl}[\mathrm{M}+\mathrm{H}]+207.0213$, found 207.0217.

\section{Acknowledgment}

Authors acknowledge support of this project by CNRS, University Paris Sud, and by La Ligue Nationale Contre le Cancer through an Equipe Labellisée 2014 grant. Guangkuan Zhao and Ling-Zhi Yuan thank the CSC (Chinese Scholarship Council) for their Ph.D. fundings. Our laboratory BioCIS-UMR 8076 is a member of the Laboratory of Excellence LERMIT supported by a grant from the Agence Nationale de la Recherche, (ANR10-LABX-33). 


\section{Supporting Information}

YES (this text will be updated with links prior to publication)

\section{Primary Data}

NO (this text will be deleted prior to publication)

\section{References}

(1) Sarbani P.; Vijaykumar C.; Manojit P. Curr. Org. Chem. 2011, 15 782 .

(2) (a) Mizuno, M.; Yoshida, S.; Iinuma, M.; Tanaka, T.; Lang, F.A.; Goto, K. Chem. Pharm. Bull. 1990, 38, 2075. (b) Saeed, A.; Rafique, H.; Ashraf, Z. J. Asian Nat. Prod. Res. 2013, 15, 130. (c) Iinuma, M.; Tosa, H.; Tanaka, T.; Mizuno, M. J. Nat. Prod. 1993, 56, 1638. (d) Rama, N. H.; Iqbal, R.; Zamani, K.; Saeed, A.; Hussain, M. T. J. Chem. Soc. Pak. 2000, 22, 130

(3) Napolitano, E. Org. Prep. Proced. Int 1997; 631.

(4) Saeed, A.; Mahesar, P. A. Tetrahedron 2014, 70, 1401.

(5) Saeed; A.; Rama, N.H.; Arfan, M. J. Heterocyclic Chem. 2003, 40, 519

(6) Kale, A.P.; Pawar, G.G.; Kapur, M. Org. Lett. 2012, 14, 1808.

(7) (a) Tréguier, B.; Rasolofonjatovo, E.; Hamze, A.; Provot, O.; Bignon, J.; Dubois, J.; Brion, J.-D.; Alami, M. Eur.J. Org. Chem. 2011, 4868. (b) Lawson, M. Hamze, A.; Peyrat, J.-F.; Bignon, J.; Dubois, J.; Brion, J.D.; Alami, M. Org. Biomol. Chem. 2013, 11, 3664.
(8) (a) Jacubert, M.; Hamze, A.; Provot, 0.; Peyrat, J.-F.; Brion, J.-D. Alami Tetrahedron Lett. 2009, 50, 3588. (b) Jacubert, M.; Provot, O.; Peyrat, J.-F.; Hamze, A.; Brion, J.-D.; Alami Tetrahedron 2010, 66, 3775. (c) Mousset, C.; Provot, O.; Hamze, A.; Bignon, J.; Brion, J.-D.; Alami, M. Tetrahedron 2008, 64, 4287. (d) Treguier, B.; Rasolofonjatovo, E.; Hamze, A.; Provot, O.; Wdzieczak-Bakala, J.; Dubois, J.; Brion, J.-D.; Alami, M. Eur. J. Org. Chem. 2011, 4868. (e) Rasolofonjatovo, E.; Treguier, B.; Provot, O.; Hamze, A.; Morvan, E.; Brion, J.-D.; Alami, M. Tetrahedron Lett. 2011, 52, 1036. (f) Rasolofonjatovo, E.; Treguier, B.; Provot, O.; Hamze, A.; Brion, J.-D.; Alami, M. Eur. J. Org. Chem. 2012, 1603. (g) Rasolofonjatovo, E.; Provot, O.; Hamze, A.; Rodrigo, J.; Bignon, J.; Wdzieczak-Bakala, J.; Lenoir, C.; Desravines, D.; Dubois, J.; Brion, J.-D.; Alami, M. Eur. J. Med. Chem. 2013, 62, 28. (h) Renko, D.; Provot, O.; Rasolofonjatovo, E.; Bignon, J.; Rodrigo, J.; Dubois, J.; Brion, J.-D.; Hamze, A.; Alami, M. Eur. J. Med. Chem. 2015, 90, 834.

(9) Le Bras G.; Hamze, A.; Messaoudi, S.; Provot, O.; Le Calvez, P.B.; Brion, J.-D.; Alami, M. Synthesis 2008, 1607.

(10) (a) Alami, M.; Gueugnot, S.; Domingues, E.; Linstrumelle, G. Tetrahedron 1995, 51, 1209. (b) Alami, M.; Peyrat, J.-F.; Brion, J.-D. Synthesis 2000, 1499.

(11) Tikad, A. Hamze, A.; Provot, O.; Brion, J.-D.; Alami, M. Eur. J. Org. Chem. 2010, 725.

(12) Baldwin, J. E. J. Chem. Soc., Chem. Commun. 1976, 734

(13) Littke, A. F.; Dai, C.; Fu, G. C. J. Am. Chem. Soc. 2000, 122, 4020.

(14) Penthala, N.R.; Thakkar, S.; Crooks, P.A. Bioorg. Med. Chem. Lett. 2015, 25, 2763. 